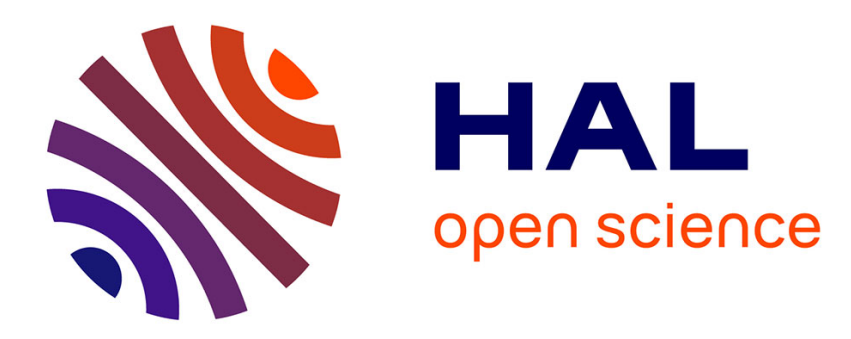

\title{
Impulsive systems and behaviors in the theory of linear dynamical systems
}

\author{
Henri Bourlès
}

\section{To cite this version:}

Henri Bourlès. Impulsive systems and behaviors in the theory of linear dynamical systems. Forum Math, 2005, 17, pp.781 - 807. hal-02368177

\section{HAL Id: hal-02368177 \\ https://hal.science/hal-02368177}

Submitted on 18 Nov 2019

HAL is a multi-disciplinary open access archive for the deposit and dissemination of scientific research documents, whether they are published or not. The documents may come from teaching and research institutions in France or abroad, or from public or private research centers.
L'archive ouverte pluridisciplinaire HAL, est destinée au dépôt et à la diffusion de documents scientifiques de niveau recherche, publiés ou non, émanant des établissements d'enseignement et de recherche français ou étrangers, des laboratoires publics ou privés. 


\title{
Impulsive systems and behaviors in the theory of linear dynamical systems
}

\author{
Henri Bourlès \\ (Communicated by Michel Fliess)
}

\begin{abstract}
A linear dynamical system resulting from the interconnection of subsystems is considered. Assuming that this interconnection is "temporal", i.e. starting at a given initial time in the continuous-time case and ending at a given final time in the discrete-time case, such a system is also said to be "temporal". Temporal interconnections generate "uncontrollable impulsive behaviors" which are not found in the classical theory, though they have been studied for more than 20 years in the case of systems with constant coefficients. Determining the structure of the impulsive behavior of a temporal system is a key problem in the theory of linear dynamical systems. It is addressed here, using module theory, for systems with time-varying coefficients, in both the continuous- and discrete-time cases. These two cases are merged into a general framework. The impulsive behavior of a temporal system satisfying a suitable regularity condition has a structure which is fully elucidated. It turns out that the determination of this structure in practice is an algebraic - not an analytic-problem, which makes the calculations simpler and easier to computerize. The theory is illustrated through several examples.
\end{abstract}

2000 Mathematics Subject Classification: 93.

\section{Introduction}

Continuous- or discrete-time systems exhibit "impulsive motions", i.e., in the continuous-time case, linear combinations of the Dirac distribution $\delta$ and its derivatives [35], [36], and in the discrete-time case, backward solutions with finite support [21], [24]. The space spanned by all impulsive motions of a system is called its "impulsive behavior" and is denoted as $\mathscr{B}_{\infty}$. The purpose of this paper is to study the structure of $\mathscr{B}_{\infty}$, for a system with constant or time-varying coefficients. Let us explain the importance of this structure.

Consider the following continuous-time system with constant coefficients, in "descriptor form" [32]:

$$
(E \partial-A) x=B u, \quad t \in \mathbb{T}_{0}
$$

where the function $u$ is the "system input", assumed to be known, $x$ is the "descriptor vector" and $\partial$ is the "continuous-time derivative", i.e. the distributional derivative 
with respect to time $t$; $E, A$ and $B$ are matrices belonging to $\Re^{q \times q}, \Re^{q \times q}$ and $\Re^{q \times m}$, respectively. Suppose that $\mathbb{T}_{0}=[0,+\infty[$, which means that the system is formed at time $t=0$ (as a result, for example, of switching or of component failure in some other system [35]; such events are frequent in electrical circuits, mechanics, hydraulics, etc. [13]). Therefore, let us call (1) a "temporal system" (to point out the difference with the classic situation where $\mathbb{T}_{0}=\Re$, and where system (1) is thus perpetually existing). Assume that the matrix pencil $E s-A$ is regular (i.e. that the polynomial $|E s-A|$ is nonzero [12]) for (1) to have solutions [18]. If $E$ is singular, the restrictions to $\mathbb{T}_{0}$ of the components $x_{i}$ of $x$ contain impulsive motions with coefficients only depending on the "initial values" $x_{i}\left(0^{-}\right)$, when the latter are incompatible with the equation $(E \partial-A) x=B u$. These impulsive motions, which are said to be "uncontrollable" due to their complete dependence on initial conditions, span the "uncontrollable part" $\mathscr{B}_{\infty, u}$ of $\mathscr{B}_{\infty}$. To know what event arose at time $t=0$, the values of the above-mentioned coefficients are not significant, as opposed to the structure of $\mathscr{B}_{\infty, u}$. Setting $\mathbb{T}=\Re$, the temporal system (1) can be written in the more general form

$$
\left\{\begin{array}{l}
B(\partial) w(t)=e(t), \quad t \in \mathbb{T} \\
e(t)=0, \quad t \in \mathbb{T}_{0}
\end{array}\right.
$$

where $B(\partial)$ is a $q \times k$ matrix $(k=q+m)$ with entries in $\Re[\partial]$ and $w$ is the columnmatrix whose entries are the system variables (here the components $x_{i}$ and $u_{i}$ of $x$ and $u$, respectively); the function $e$ has any restriction to the complement $\mathbb{T} \backslash \mathbb{T}_{0}$ of $\mathbb{T}_{0}$ in $\mathbb{T}$. It is known that the structure of $\mathscr{B}_{\infty, u}$ is completely determined by the structure of the "zeros at infinity" of the matrix $B(\partial)$ ([33], [18]) - a notion which is explained below. Therefore, the characterization of the structure of $\mathscr{B}_{\infty, u}$ is not an analytic problem (involving derivations, integrations, etc., in the framework of the theory of distributions), but an algebraic one, which makes the calculations much simpler and easier to computerize.

A similar problem is posed by discrete-time systems [21], [22]. The variables are now sequences (denoted as functions defined on the set of integers $\mathbb{Z}$ ). Let $\boldsymbol{q}$ be the usual "shift forward operator" $w(t) \rightarrow w(t+1)$, define the "discrete-time derivative" $\partial=\boldsymbol{q}-1$, and with this notation consider the discrete-time system with constant coefficients (1). Assume that the sequence $u$ (again called the "input") is known and that the matrix pencil $E z-A$ is regular. Suppose that the matrix $E$ is singular (which means that the system is noncausal) and that $\mathbb{T}_{0}=\{\ldots,-2,-1,0\}$, i.e. that the system exists only up to the "final time" $t=0$ (a phenomenon which arises in various fields: for example the "Leontief model", in economy, describes the time pattern of production in several interrelated production sectors; it is of the form (1), possibly noncausal, and valid up to a finite final time [23]). For the same reason as above, let us call (1) (or (2) which is the most general form) a "temporal system". Due to the fact that (1) is noncausal, the restrictions to $\mathbb{T}_{0}$ of the variables $x_{i}$ contain backward solutions with finite support (i.e. impulsive motions), with coefficients only depending on the "final values" $x_{i}(1)$. As in the continuous-time case, these impulsive motions, said to be uncontrollable due to their complete dependence on final conditions, span the "uncontrollable part" $\mathscr{B}_{\infty, u}$ of $\mathscr{B}_{\infty}$. Considering the temporal system (2), where 
$\mathbb{T}=\mathbb{Z}$ and where the sequence $e$ has any restriction to $\mathbb{T} \backslash \mathbb{T}_{0}$, the structure of $\mathscr{B}_{\infty, u}$ is a key problem. One can deduce from recent results of the literature that this structure is determined by the "structure at infinity" of the matrix $B(\partial)([1],[16],[17])$; more specifically, it is shown below that the structure of $\mathscr{B}_{\infty, u}$ reflects the structure of the zeros at infinity of $B(\partial)$, exactly as in the continuous-time case.

In the existing literature, only the case of systems with constant coefficients has been treated, and two distinct theories have been developed to prove the abovementioned connection between the structure of $\mathscr{B}_{\infty, u}$ and that of the zeros at infinity of $B(\partial)$ : one for the continuous-time, using the Laplace transform, and the other for the discrete-time, using the Z-transform. In both cases, complicated calculations yield a very simple structure theorem, without really explaining it. These calculations become inextricable in the case of systems with time-varying coefficients, although these systems raise the same problem ([24], [6], [38]); therefore, no general result on the structure of impulsive behaviors has been obtained in that case.

The problem of determining the structure of the impulsive behavior $\mathscr{B}_{\infty}$ (and of its uncontrollable part $\mathscr{B}_{\infty, u}$ ) of a linear temporal system with constant or time-varying coefficients is solved here using an algebraic approach based on module theory. The continuous- and discrete-time cases are merged into a general framework. In the case of constant coefficients, complicated calculations are avoided, and the existing theory is both clarified and completed. In the case of time-varying coefficients, one difficulty arises from singularities which may occur, typically whenever a system coefficient annihilates a part of an impulsive motion when vanishing (e.g., in the continuous-time case, an impulsive motion proportional to $\delta$ is annihilated by a nonzero coefficient $a$ such that $a(0)=0)$. A temporal system with no such problem is said to be "regular". We show that, for regular temporal systems, the structure of $\mathscr{B}_{\infty, u}$ is still completely determined by the structure of the zeros at infinity of the matrix $B(\partial)$ (once this notion has suitably been generalized [5], [25]).

The notion of "temporal interconnection" is useful for the sequel. Any system may be considered as resulting from the interconnection of subsystems [30]. In the continuous-time case, a switching, a component failure, etc., as mentioned above, are interconnections starting at a given initial time (assumed to be zero without loss of generality, since the origin of time can be freely chosen), i.e. only effective on $\mathbb{T}_{0}=$ $[0,+\infty[\subset \mathbb{T}$; such an interconnection is said to be "temporal" in what follows. In the discrete-time case, a temporal interconnection is an interconnection valid up to a given final time (also assumed to be zero), i.e. only effective on $\mathbb{T}_{0}=\{\ldots,-2,-1,0\} \subset \mathbb{T}$. A temporal system results from the temporal interconnection of subsystems. This is clear when considering (2) which is obtained by interconnecting the system $B(\partial) w=e$ with the trivial system $\bar{e}=0$ through the temporal interconnection $e(t)=\bar{e}(t), t \in \mathbb{T}_{0}$.

The paper is organized as follows. Preliminaries are collected in Section 2: first the mathematical tools, and then the basic notions of system theory. Temporal systems with constant coefficients (case (I)) are studied in Section 3: using a key isomorphism, it is shown that the structure of $\mathscr{B}_{\infty}$ is identical to the structure of a space $\mathscr{A}_{\infty}$, easier to study than $\mathscr{B}_{\infty}$, and whose construction is classic in homological algebra. The space $\mathscr{A}_{\infty}$ is also studied in Section 4 in the case when the system coefficients are timevarying though belonging to a field (case (II)). The assumption that the coefficients 
belong to a field, amounts to discarding singularities (since coefficients which are not identically zero cannot vanish). However, to properly define and study the impulsive behavior $\mathscr{B}_{\infty}$ of a temporal system with time-varying coefficients, one must assume that these coefficients belong to a ring of functions (case (III)). This case is studied in Section 5. Most of the results in Section 3 are extended to case (III) under a suitable regularity condition. Section 6 includes the concluding remarks and summarizes the main results. Preliminary results have already been published [2], [3].

\section{Preliminaries}

\subsection{Some algebra}

\subsubsection{Differential polynomials and formal power series}

A general framework. Using general differential polynomials, one can study continuous- and discrete-time systems with constant or time-varying coefficients in a unique framework.

Consider first the continuous-time case. Let $\boldsymbol{K}$ be a commutative Noetherian domain equipped with the continuous-time derivative $a \rightarrow \dot{a}=\frac{d a}{d t}(a \in \boldsymbol{K})$, which is assumed to be an endomorphism of $\boldsymbol{K} ; \boldsymbol{K}$ is the ring to which the coefficients of the system under study belong (and is called the "coefficient ring", for short). The elements of $\boldsymbol{K}[\partial]$ (where $\partial$ is the indeterminate introduced in Section 1) are operators on the system variables. Let $w$ be such a variable and $a \in \boldsymbol{K}$. From the Leibniz rule: $\partial(a w)=a \partial w+\dot{a} w$; this yields the "commutation rule"

$$
\partial a=a \partial+\dot{a} .
$$

Consider now the discrete-time case. The coefficient ring $\boldsymbol{K}$ is a commutative Noetherian domain equipped with the derivative $a \rightarrow a^{\gamma}=a^{\alpha}-a$, where $a^{\alpha}(t)=$ $a(t+1)$, assuming that $\alpha$ is an automorphism of $\boldsymbol{K}$. Let $w$ be a system variable and consider the indeterminate $\partial=\boldsymbol{q}-1$, as in Section 1. One has [15]: $\partial(a w)(t)=$ $a(t+1) w(t+1)-a(t) w(t)=a(t+1)(w(t+1)-w(t))+(a(t+1)-a(t)) w(t)$, which yields the commutation rule

$$
\partial a=a^{\alpha} \partial+a^{\gamma}
$$

A derivation $\gamma$ for which the commutation rule (4) holds is called an " $\alpha$-derivation" [7]. Clearly, (3) is of the form (4) with $\alpha=1$ (i.e. identity). Thus, the continuous- and discrete-time cases are merged into a unique general framework, assuming that $\boldsymbol{K}$ is a commutative Noetherian domain equipped with an $\alpha$-derivation $\gamma$ where $\alpha$ is an automorphism of $\boldsymbol{K}$. The subring of constants of $\boldsymbol{K}$ (consisting of all elements $a$ such that $\left.a^{\gamma}=0\right)$ is denoted as $\boldsymbol{k}$; in everything that follows, $\boldsymbol{k}$ is a field, and, except when explicitly stated, "space" means " $\boldsymbol{k}$-vector space".

The ring of differential polynomials with coefficients in $\boldsymbol{K}$ and indeterminate $\partial$, equipped with the commutation rule (4), is denoted by $\boldsymbol{K}[\partial ; \alpha, \gamma]$, as usual [7], and we set $\boldsymbol{R}=\boldsymbol{K}[\partial ; \alpha, \gamma]$. 
Three cases. Let us specify the cases considered in the sequel:

(I) $\quad \boldsymbol{K}=\Re$ (case of constant coefficients);

(II) $\boldsymbol{K}$ is a field;

(III) $\boldsymbol{K}=\Re[t]$ (in the continuous- or discrete-time case) or $\boldsymbol{K}=\Re\{t\}$ (in the continuous-time case only). The ring $\Re[t]$ is identified with the ring of polynomial functions on the real line, and $\Re\{t\}$ denotes the ring of convergent power series with real coefficients.

Cases (I), (II) and (III) are studied in Sections 3, 4 and 5, respectively.

Formal power and Laurent series. Set $\sigma=1 / \partial$ and $\beta=\alpha^{-1} ; \boldsymbol{S}:=\boldsymbol{K}[[\sigma ; \beta, \gamma]]$ denotes the ring of formal power series in $\sigma$, equipped with the commutation rule [7]

$$
\sigma a=a^{\beta} \sigma-\sigma a^{\beta \gamma} \sigma
$$

deduced from (4). Similarly, $\boldsymbol{L}=\boldsymbol{K}((\sigma ; \beta, \gamma))$ is the ring of formal Laurent series in $\sigma$, equipped with the commutation rule (5). The rings $\boldsymbol{R}$ and $\boldsymbol{S}$ can be embedded in $\boldsymbol{L}=\boldsymbol{K}((\sigma ; \beta, \gamma))$; all these rings are domains (i.e. integral rings) and are noncommutative, except if $\boldsymbol{K}=\boldsymbol{k}$. As $\sigma \boldsymbol{S}=\boldsymbol{S} \sigma$, this two-sided ideal is denoted by $(\sigma)$; the units of $\boldsymbol{S}$ are the power series whose constant term is a unit of $\boldsymbol{K}$. The ring $\boldsymbol{L}$ is obtained from $\boldsymbol{R}$ by "localization at infinity" (which yields $\boldsymbol{K}\left[\partial, \partial^{-1} ; \alpha, \gamma\right]$, the ring of skew Laurent polynomials [26]) and then "completion at infinity" (i.e. completion with respect to the $(\sigma)$-adic topology).

Properties of the rings. The domains $\boldsymbol{R}$ and $\boldsymbol{S}$ are Noetherian since so is $\boldsymbol{K}$ ([26], $\S \S 1.2 .9,1.4 .5)$, therefore they are Ore $([7], \S 0.8)^{1}$. In case (III), one has the following result:

Lemma 1. Let us assume that $\boldsymbol{K}=\Re[t]$. (i) Any element of $\boldsymbol{L}$ is of the form $\sum_{i \geq 0 \text {, finite }} b_{i}(\sigma) t^{i}, b_{i}(\sigma) \in \Re((\sigma))$, i.e. $\boldsymbol{L}$ is a polynomial ring with coefficients in $\Re((\sigma))$. (ii) Setting $\xi=0$ in the continuous-time case and $\xi=1$ in the discrete-time one, $\boldsymbol{L}$ is equipped with the commutation rule $t a=a t+a^{\varepsilon}$, where $a \in \Re((\sigma))$ and $a^{\varepsilon}:=$ $-(\xi \partial+1) \frac{d a}{d \partial}$. (iii) The ring $\boldsymbol{L}=\Re((\sigma))[t ; 1, \varepsilon]$ is a simple principal ideal domain.

Proof. (i) is obvious. (ii): By induction, for any $i \in \mathbb{Z}, t \partial^{i}=\partial^{i} t-(\xi \partial+1) i \partial^{i-1}=$ $\partial^{i} t-(\xi \partial+1) \frac{d\left(\partial^{i}\right)}{d \partial}$. Using (i), it is easy to obtain (ii). (iii): As $\varepsilon$ is a 1-derivation of the field $\Re((\sigma)), \boldsymbol{L}=\Re((\sigma))[t ; 1, \varepsilon]$ is a principal ideal domain ([7], §8.3). An element $f$ of $\boldsymbol{L}$ is right invariant if, and only if properties (a) and (b) below are satisfied ([7], §8.3, Proposition 3.2): (a) $t f=f t$, which implies $f \in \Re[t]$, according to the commutation

\footnotetext{
${ }^{1}$ In what follows, "noncommutative" means "possibly noncommutative". In addition, and with this understanding, "field" means "noncommutative field" (also called "skew field" or "division ring" by many authors), "Ore" means "left and right Ore", "Noetherian" means "left and right Noetherian", etc.
} 
rule in (ii). (b) For any $c \in \Re((\sigma))$, $c f=f c$; with $c=\sigma$, this implies $f \in \Re$ by (5). Therefore, $\boldsymbol{L}$ is simple ([7], §8.3, Corollary 3.6).

In cases (I) and (II), $\boldsymbol{R}$ and $\boldsymbol{S}$ are principal ideal domains, $\boldsymbol{S}$ is local with maximal ideal $(\sigma)$, and $\boldsymbol{L}$ is the quotient field of $\boldsymbol{S}$. All these rings are commutative in case $(\mathrm{I})$. $\boldsymbol{k}=\Re$ in cases (I) and (III).

\subsubsection{Modules}

Let $\boldsymbol{D}$ be a Noetherian domain with quotient field $\boldsymbol{Q} ; \boldsymbol{D} \mathscr{M}\left(\operatorname{resp} . \boldsymbol{D} \mathscr{M}^{f}\right)$ denotes the category of all left (resp. finitely generated left) $\boldsymbol{D}$-modules. Due to the Noetherian property of $\boldsymbol{D}$, any $M \in \boldsymbol{D} \mathscr{M}^{f}$ is finitely presented, i.e. it has a presentation

$$
E \stackrel{f}{\rightarrow} F \stackrel{\phi}{\rightarrow} M \rightarrow 0
$$

where, e.g., $E=\boldsymbol{D}^{q}, F=\boldsymbol{D}^{k}(q \leq k)$ and where $\phi: F \rightarrow$ coker $f$ is the canonical epimorphism. Let $M$ be defined by (6), let $\left(\varepsilon_{i}\right)_{1 \leq i \leq q}$ and $\left(\underline{\mathrm{w}}_{i}\right)_{1 \leq i \leq k}$ be bases of $E$ and $F$, respectively, and assume that the elements of $E$ and $F$ are represented by rowmatrices in those bases, as usual in the theory of "D-modules" [14]. Let $B \in \boldsymbol{D}^{q \times k}$ be the matrix representing $f$; $f$ is the right multiplication by $B$ (written $\bullet B$ in the literature). Setting $e_{i}=f\left(\varepsilon_{i}\right), 1 \leq i \leq q$, one has $e_{i}=\varepsilon_{i} B$, thus

$$
B \underline{\mathrm{w}}=e
$$

where $e=\left[e_{1}, \ldots, e_{q}\right]^{T}$ and $\underline{\mathrm{w}}=\left[\underline{\mathrm{w}}_{1}, \ldots, \underline{\mathrm{w}}_{k}\right]^{T}$. The module $M=$ coker $\bullet B$ (i.e. the module with "matrix of definition" $B)$ is generated by the elements $w_{i}=\phi\left(\underline{\mathrm{w}}_{i}\right)$, $1 \leq i \leq k\left(\right.$ written $M=[w]_{\boldsymbol{D}}$, where $\left.w=\left[w_{1}, \ldots, w_{k}\right]^{T}\right)$, such that

$$
B w=0 .
$$

This module $M$ is said to be defined by generators (the elements $\underline{\mathrm{w}}_{i}$ ) and relations (the rows of (8)) [29].

As $\boldsymbol{D}$ is an Ore domain, the set of torsion elements of $M$ is a submodule of $M$ [7].

\subsubsection{Matrices}

Completely left coprime factorizations. Let $V$ be a matrix with entries in $\boldsymbol{D}$ (or "a matrix over D", for short). Assume that $V$ is right regular; $V$ is said to be completable if there exists a matrix $W$ over $\boldsymbol{D}$, having the same number of columns as $V$, such that $\left[\begin{array}{c}V \\ W\end{array}\right]$ is unimodular [7]. Left coprimeness of matrices over $\boldsymbol{D}$ is not an ambiguous notion when $\boldsymbol{D}$ is a principal ideal domain. In more general cases, several kinds of left coprimeness may be defined. In this paper, the following notion will be useful: 
Definition 2. Let $D$ and $N$ be two matrices over $\boldsymbol{D}$, having the same number of rows, and assume that $V:=\left[\begin{array}{ll}D & N\end{array}\right]$ is right regular. The pair $(D, N)$ is said to be completely left coprime if $V$ is completable.

Let $\hat{B} \in \boldsymbol{Q}^{q \times k}$. There exist matrices $D \in \boldsymbol{D}^{q \times q}$ and $N \in \boldsymbol{D}^{q \times k}$ such that $D$ is of rank $q$ (i.e. invertible over $\boldsymbol{Q})^{2}$ and $\hat{B}=D^{-1} N$.

Definition 3. The above pair $(D, N)$ is said to be a completely left coprime factorization $(C L C F)$ of $\hat{B}$ over $\boldsymbol{D}$, if this pair is completely left coprime.

Remark 4. As is well known, a left coprime factorization of any matrix $\hat{B} \in \boldsymbol{Q}^{q \times k}$ over $\boldsymbol{D}$ exists if $\boldsymbol{D}$ is a principal ideal domain, as a consequence of the Smith form ([7], Chap. 8). If $\boldsymbol{D}$ is more general, e.g., a Noetherian domain, a completely left coprime factorization of $\hat{B}$ over $\boldsymbol{D}$ does not necessarily exist.

Smith-MacMillan form at infinity. Let us consider the rings $\boldsymbol{R}$ and $\boldsymbol{S}$, as defined in $\$ 2.1 .1$, and let $B(\partial) \in \boldsymbol{R}^{q \times k}$ be a matrix of rank $r$. The following result is classic in case (I) [32] and has been extended to case (II) in [5]: there exist two unimodular matrices $U(\sigma) \in \boldsymbol{S}^{q \times q}$ and $V(\sigma) \in \boldsymbol{S}^{k \times k}$, as well as integers $v_{1}, \ldots, v_{r}, v_{1} \leq \cdots \leq v_{r}$, such that

$$
U(\sigma) B(\partial) V^{-1}(\sigma)=\left[\begin{array}{cc}
\operatorname{diag}\left\{\sigma^{v_{i}}\right\}_{1 \leq i \leq r} & 0 \\
0 & 0
\end{array}\right],
$$

The matrix in the right-hand member of (9) is called the Smith-MacMillan form at infinity of $B(\partial)$ ([34], [32]). Define the finite sequences $\left(\bar{\mu}_{i}\right)_{1 \leq i \leq r}$ and $\left(\bar{\pi}_{i}\right)_{1 \leq i \leq r}$ as: $\bar{\mu}_{i}=\max \left(0, v_{i}\right)$ and $\bar{\pi}_{i}=\max \left(0,-v_{i}\right)$. Among the integers $\bar{\mu}_{i}\left(\right.$ resp. $\left.\bar{\pi}_{i}\right)$, those which are nonzero (if any) are called the structural indexes of the zeros at infinity (resp. of the poles at infinity) of the matrix $B(\partial)$ ([5], [25]); they are put in increasing (resp. decreasing) order and denoted by $\mu_{i}(1 \leq i \leq \rho)\left(\right.$ resp. $\left.\pi_{i}(1 \leq i \leq s)\right)$.

\subsubsection{Duality}

Kernels. Let $\boldsymbol{D}$ be the ring $\boldsymbol{R}$ or $\boldsymbol{S}$ in $\$ 2.1 .1 ; \boldsymbol{D}$ is a $\boldsymbol{k}$-algebra. Let $W \in \boldsymbol{D} \mathscr{M}$, and let $M \in \boldsymbol{D} \mathscr{M}^{f}$ be the module presented by (6), i.e. $M=$ coker $\bullet B$. The abelian group $\operatorname{Hom}_{\boldsymbol{D}}(M, W)$ consisting of all homomorphisms $M \rightarrow W$ has a canonical structure of $\boldsymbol{k}$-vector space and of left $\boldsymbol{E}$-module, where $\boldsymbol{E}$ is the endomorphism ring of $W$, since $W$ is a left $(\boldsymbol{E}, \boldsymbol{D})$-bimodule ${ }^{3} \operatorname{Hom}_{\boldsymbol{D}}(M, W)$ is called the " $W$-dual" 4 of $M$

\footnotetext{
${ }^{2}$ Recall that over an Ore domain $\boldsymbol{D}$, the row and column ranks of a matrix are equal and coincide with the rank of this matrix over the quotient field $\boldsymbol{Q}$ : see, e.g., ([7], §5.4, exerc. 11).

${ }^{3}$ An $(\boldsymbol{E}, \boldsymbol{D})$-bimodule is a left $\boldsymbol{E}$-module which is a right $\boldsymbol{D}$-module (with an associative law relating the two actions). In this paper, where all modules are left modules a "left $(\boldsymbol{E}, \boldsymbol{D})$ bimodule" is a left $\boldsymbol{E}$-module which is a left $\boldsymbol{D}$-module provided that the rings $\boldsymbol{E}$ and $\boldsymbol{D}$ are compatible, i.e. such that $e d=d e, \forall e \in \boldsymbol{E}, \forall d \in \boldsymbol{D}$ (with an obvious associative law).

${ }^{4}$ It should not be confused with the "algebraic dual" $\operatorname{Hom}_{\boldsymbol{D}}(M, \boldsymbol{D})$, which will not be used in this paper.
} 
([19], §19D) and is denoted as $M^{*}$. This module is $\boldsymbol{E}$-isomorphic to the set of all elements $w \in W^{k}$ such that $B w=0$, and is identified with this set assuming that the generators chosen for $M$ are those in $\$ 2.1 .2$. Therefore, $M^{*}$ is the kernel in $W^{k}$ of the left multiplication by $B$ (written $B \bullet$ in the literature); denoting this kernel as ker $B \bullet$, one can write $M^{*}=\operatorname{ker} B \boldsymbol{\bullet}$. In what follows, the class of all $\boldsymbol{E}$-modules of the form $M^{*}$, where $M \in \boldsymbol{D} \mathscr{M}^{f}$, is denoted as $\left(\boldsymbol{D} \mathscr{M}^{f}\right)^{*}$.

The module $\tilde{\boldsymbol{\Delta}}$. Let $\boldsymbol{D}=\boldsymbol{S}$ in any case considered in $\$ 2.1 .1$. For any $\mu \in \mathbb{N}$ (where $\mathbb{N}$ denotes the set of natural integers), set $\tilde{C}_{\mu}=\frac{\boldsymbol{s}}{\left(\sigma^{\mu}\right)}\left(\right.$ thus $\left.\tilde{C}_{0}=0\right)$ and let $\tilde{\delta}^{(\mu-1)}$ be the canonical image of $1 \in \boldsymbol{S}$ in $\tilde{C}_{\mu}$. The $\boldsymbol{S}$-module $\tilde{C}_{\mu}$ is isomorphic to a submodule of $\tilde{C}_{\mu+1}$, under right multiplication by $\sigma$, and $\tilde{\delta}^{(\mu)} \sigma=\sigma+\left(\sigma^{\mu+1}\right)=\sigma \tilde{\delta}^{(\mu)}$; identifying $\tilde{\delta}^{(\mu-1)}$ with $\sigma \tilde{\delta}^{(\mu)}, \tilde{C}_{\mu}$ is embedded in $\tilde{C}_{\mu+1}$, and

$$
\tilde{C}_{\mu}=\bigoplus_{i=1}^{\mu} \boldsymbol{K} \tilde{\delta}^{(i-1)} .
$$

Set

$$
\tilde{\Delta}:=\lim _{\vec{\mu}} \tilde{C}_{\mu}=\bigoplus_{\mu \geq 0} \boldsymbol{K} \tilde{\delta}^{(\mu)}
$$

The left $\boldsymbol{S}$-module $\tilde{\Delta}$ becomes a left $\boldsymbol{L}$-module, setting $\sigma^{-1} \tilde{\boldsymbol{\delta}}^{(\mu)}=\tilde{\boldsymbol{\delta}}^{(\mu+1)}$, and thus a left $\boldsymbol{R}$-module by restriction of the ring of scalars. Considering $\sigma$ and $\partial$ as operators on $\tilde{\Delta}$, $\sigma$ is a left inverse of $\partial$, but $\sigma$ has no left inverse since $\sigma \tilde{\delta}=0$.

Assuming that $\boldsymbol{K}$ is a field (case (II)), the only simple $\boldsymbol{S}$-module is $(\sigma)$, and the $\boldsymbol{S}$ module $\tilde{\Delta}$ is the canonical cogenerator of $\boldsymbol{s} \mathscr{M}([19], \S 3)$.

Assuming that $\boldsymbol{K}=\Re$ (case (I)), $\boldsymbol{S}$ is commutative. According to Matlis' theory ([19], §3I), as $\boldsymbol{S}$ is complete (for the $(\sigma)$-adic topology), $\boldsymbol{S}$ and the endomorphism ring $\boldsymbol{E}$ of $\tilde{\Delta}$ are isomorphic (as rings), thus these two rings are identified.

A useful lemma. In the lemma below, $\boldsymbol{D}$ is a Noetherian domain, $W$ is a $\boldsymbol{D}$-module and $\boldsymbol{E}$ is the endomorphism ring of $W$.

Lemma 5. (i) Consider the following relation, denoted as $\simeq$, between two elements of $\left(\boldsymbol{D} \mathscr{M}^{f}\right)^{*}: M^{\prime} \simeq N^{\prime}$ if (and only if) there exist two $\boldsymbol{D}$-modules $M$ and $N$ in $\boldsymbol{D} \mathscr{M}^{f}$ such that $M^{\prime}=M^{*}, N^{\prime}=N^{*}$ and $M \cong \boldsymbol{D} N$; it is an equivalence relation, and $M^{\prime} \simeq N^{\prime}$ implies $M^{\prime} \cong \boldsymbol{E} N^{\prime}$. (ii) Let $d \in \boldsymbol{D}$ be such that $d \boldsymbol{D}=\boldsymbol{D} d$, and let $M \in \boldsymbol{D} \mathscr{M}^{f}$. The set $d M$ is a submodule of $M$ and $d M^{*}=(d M)^{*}$. (iii) Let $d$ be as in (ii) and $M \in \boldsymbol{D} \mathscr{M}^{f}$. If $M$ is such that $d M=0$, then $d M^{*}=0$. Conversely, assuming that $W$ is a cogenerator, if $d M^{*}=0$, then $d M=0$. (iv) Let $0 \neq d$ be as in (ii), and let $n$ and $m$ be natural integers such that $M^{*} \simeq W^{m}, N^{*} \simeq W^{n}$ and $d M^{*}=d N^{*}$; if $W$ is a cogenerator, then $M^{*}=N^{*}$.

Proof. (i): The relation $\simeq$ is obviously an equivalence relation. Assuming that $M^{*} \simeq N^{*}$, there exists a $\boldsymbol{D}$-isomorphism $j: M \stackrel{\sim}{\rightarrow} N$. Let $j^{*}: N^{*} \rightarrow M^{*}$ be defined 
as: $j^{*} \lambda:=\lambda j, \lambda \in N^{*} ; j^{*}$ is an $\boldsymbol{E}$-isomorphism. (ii): As $d \boldsymbol{D}=\boldsymbol{D} d, d M$ is an $\boldsymbol{S}$ submodule of $M$. Let $\lambda \in(d M)^{*}$; for any $m \in M, \lambda(d M)=d \lambda(m)=(d \lambda)(m)$. The mapping $(d M)^{*} \ni \lambda \rightarrow d \lambda \in d M^{*}$ is a canonical $\boldsymbol{E}$-isomorphism under which the $\boldsymbol{E}$ modules $(d M)^{*}$ and $d M^{*}$ are identified. (iii) is an obvious consequence of (ii). (iv): There exist two free $\boldsymbol{D}$-modules $\Phi_{m} \cong{ }_{\boldsymbol{D}} \boldsymbol{D}^{m}$ and $\Phi_{n}^{\prime} \cong_{\boldsymbol{D}} \boldsymbol{D}^{n}$ such $\boldsymbol{M}^{*}=\left(\Phi_{m}\right)^{*}$ and $N^{*}=\left(\Phi_{n}^{\prime}\right)^{*}$. By (ii), $d\left(\Phi_{m}\right)^{*}=\left(d \Phi_{m}\right)^{*}$ and $d\left(\Phi_{n}^{\prime}\right)^{*}=\left(d \Phi_{n}^{\prime}\right)^{*}$; therefore, assuming that $d M^{*}=d N^{*}$, one obtains $\left(d \Phi_{m}\right)^{*}=\left(d \Phi_{n}\right)^{*}$, which implies that $d \Phi_{m}=d \Phi_{n}^{\prime}$ if $W$ is a cogenerator $([19],(19.45))$. In addition, $d \Phi_{m} \cong \boldsymbol{D} \Phi_{m}$ and $d \Phi_{n} \cong \boldsymbol{D} \Phi_{n}^{\prime}$ (under multiplication by $d$ ), thus $\Phi_{m} \cong \boldsymbol{D} \Phi_{n}^{\prime}$, which implies $n=m$ since any Noetherian domain has invariant basis number. Thus, there exist two unimodular matrices $U$ and $V$ belonging to $\boldsymbol{D}^{n \times n}$ such that $\Phi_{n}=U \boldsymbol{D}^{n}$ and $\Phi_{n}^{\prime}=V \boldsymbol{D}^{n}$, hence $d U \boldsymbol{D}^{n}=$ $d V \boldsymbol{D}^{n}$. Therefore, $U \boldsymbol{D}^{n}=V \boldsymbol{D}^{n}$ since $\boldsymbol{D}$ is a domain, i.e. $\Phi_{n}=\Phi_{n}^{\prime}$. This implies that $M^{*}=\left(\Phi_{n}\right)^{*}=\left(\Phi_{n}^{\prime}\right)^{*}=N^{*}$.

\subsection{Systems theory}

\subsubsection{Linear systems and their interconnections}

Smooth linear systems. According to Fliess [8], a linear system is a module $M \in \boldsymbol{R} \mathscr{M}^{f}$. It has an equation of the form

$$
B(\partial) w=0
$$

similar to (8). This is a "cokernel representation", since $M=$ coker $\bullet B(\partial)$. In the context of this paper, this module $M$ is called, more specifically, the smooth system defined by (12) (or by the matrix $B(\partial)$ ). The "module of uncontrollable poles" of $M$ (also called its "module of input-decoupling zeros" [4]) is its torsion submodule $\mathscr{T}(M)$. The system $M$ is said to be controllable if it is torsion-free [8] $]^{5}$. Considering two $\boldsymbol{R}$-submodules $M_{1}$ and $M_{2}$ of $M$, such that $M_{1} \subset M_{2}$, one has

$$
Q_{2} \simeq_{R} \frac{Q_{1}}{M_{2} / M_{1}}
$$

where $Q_{1}=M / M_{1}$ and $Q_{2}=M / M_{2}$. Therefore, the following relation among pairs of quotients of $M$ is an order relation: $Q_{2} \leq Q_{1}$ if (and only if) $Q_{2}$ is $\boldsymbol{R}$-isomorphic to a quotient of $Q_{1}$. Let $\boldsymbol{C}(M)$ be the set of all quotients of $M$ which are controllable systems, ordered by the above relation; $M / \mathscr{T}(M)$ is the greatest element of $\boldsymbol{C}(M)$.

Definition 6. The system $M / \mathscr{T}(M)$ is called the controllable quotient of $M^{6}$.

\footnotetext{
5 There are different notions of controllability [10]; we are considering here "torsion-free controllability". In cases (I) and (II) in \$2.1.1, and all kinds of controllability are equivalent since $\boldsymbol{R}$ is a principal ideal domain.

${ }^{6}$ We do not specify: the greatest controllable quotient, for short.
} 
In cases (I) and (II) in $\S 2.1 .1$, as $\boldsymbol{R}$ is a principal ideal domain, there exists a free module $\Phi$ such that

$$
M=\Phi \oplus \mathscr{T}(M)
$$

therefore $\Phi \cong \boldsymbol{R} M / \mathscr{T}(M)$.

Interconnection of smooth linear systems. The interconnection of (smooth) linear systems is defined in [9]. In the case of several systems, one may first interconnect two of them, then interconnect a third one with the system resulting from the interconnection of the two first ones, etc. Therefore, it is sufficient to consider the case of two smooth linear systems $M_{1}$ and $M_{2}$. Their interconnection is a fibered sum [20]: let $G$ be a free module in $\boldsymbol{R} \mathscr{M}^{f}$ and assume that there exist two morphisms $h_{i}: G \rightarrow M_{i}$, $i=1,2$. Let $H$ be the submodule of $M=M_{1} \oplus M_{2}$ generated by the elements of the form $h(g)=\left(h_{1}(g),-h_{2}(g)\right), g \in G$, i.e. $H=\operatorname{Im} h$, where $h=\left(h_{1},-h_{2}\right)$. The quotient module $\breve{M}=M / H$, written $M_{1} \sqcup_{G} M_{2}$, is the fibered sum of $M_{1}$ and $M_{2}$ over $G$ (with respect to the morphisms $h_{1}, h_{2}$ ); from the point of view of systems theory, it is the interconnected smooth system.

Let $\xi: M \rightarrow \breve{M}$ be the canonical epimorphism and set $\breve{h}=\xi h$, so that

$$
\breve{h}(g)=0, \quad g \in G .
$$

The system $\breve{M}$ is defined by an equation consisting of the equations of the subsystems $M_{i}$, plus the interconnection equation (14). More specifically, let us assume that $M_{i}$ is defined by the equation $B_{i}(\partial) w^{i}=0(i=1,2)$, where $w^{i}=\left(w_{1}^{i}, \ldots, w_{k_{i}}^{i}\right)$. With respect to these presentations, the interconnection equation can be written $J_{1}(\partial) \breve{w}^{1}=$ $J_{2}(\partial) \breve{w}^{2}$, where $J_{1}(\partial)$ and $J_{2}(\partial)$ are matrices over $\boldsymbol{R}$, with the same number of rows and with, respectively, $k_{1}$ and $k_{2}$ columns. Therefore,

$$
\breve{M}=\text { coker } \bullet\left[\begin{array}{cc}
B_{1}(\partial) & 0 \\
0 & B_{2}(\partial) \\
J_{1}(\partial) & -J_{2}(\partial)
\end{array}\right]
$$

(see [9] for more details).

\subsubsection{Behavioral theory}

Behaviors. In the behavioral theory [39], [28], one is interested in the solutions of (12) in a space of (generalized) functions or sequences $W ; W$ is assumed to be an $\boldsymbol{R}$ module. Let $\boldsymbol{E}$ be the endomorphism ring of $W$ and $M=\operatorname{coker} \bullet B(\partial)$; as already said, the set of all the above-mentioned solutions is an $\boldsymbol{E}$-module, written $\operatorname{ker} B(\partial) \bullet$, and identified with $M^{*}=\operatorname{Hom}_{\boldsymbol{R}}(M, W)$. This $\boldsymbol{E}$-module $\operatorname{ker} B(\partial) \bullet$ is called the behavior (or, more specifically, in the context of this paper, the smooth behavior) associated with $M$ in a product of copies of $W[39]^{7}$.

\footnotetext{
7 The terminology used in [27], [11] for the same concepts is slightly different.
} 
Generally speaking, whereas a "system" $M$ is a cokernel, the associated "behavior" $M^{*}$ is the corresponding kernel (in a product of copies of a specified "solution space" $W)$. Of course, $M^{*}$ can be determined from $M$; the converse is true if $W$ is a cogenerator since, in that case, $M^{*}=N^{*}$ implies $M=N$ ([27], (2.47); [19], (19.45)). If two behaviors $N^{*}$ and $M^{*}$ are such that $N^{*} \subset M^{*}, N^{*}$ is said to be a subbehavior of $M^{*}([27])$.

Remark 7. Let $Q=\frac{M}{N}$ be a quotient of $M$, and consider the short exact sequence

$$
0 \rightarrow N \rightarrow M \rightarrow Q \rightarrow 0
$$

As the functor $\operatorname{Hom}_{\boldsymbol{R}}(., W)$ is contravariant and left exact, it yields the exact sequence

$$
0 \rightarrow Q^{*} \rightarrow M^{*} \rightarrow N^{*}
$$

so that $Q^{*}$ can be identified with an $\boldsymbol{E}$-submodule (i.e. a subbehavior) of $M^{*}$. Assuming that $W$ is injective, one obtains the short exact sequence

$$
0 \rightarrow Q^{*} \rightarrow M^{*} \rightarrow N^{*} \rightarrow 0
$$

thus $N^{*} \cong E \frac{M^{*}}{Q^{*}}$; in other words, one has the correspondence quotient $\leftrightarrow$ submodule under $W$-duality.

A direct sum decomposition. In case (I) in $\$ 2.1 .1$, with $W=\mathscr{C}^{\infty}(\Re ; \Re)$, considering the decomposition (13), there exist subbehaviors $M_{c}^{*} \simeq \Phi^{*} \simeq(M / \mathscr{T}(M))^{*}$ and $M_{u}^{*} \simeq(\mathscr{T}(M))^{*}$ of $M^{*}$ such that

$$
M^{*}=M_{c}^{*} \oplus M_{u}^{*}
$$

The subbehavior $M_{c}^{*}$ is unique and is called the "controllable subbehavior" of $M^{*}$, whereas the subbehavior $M_{u}^{*}$ (unique up to isomorphism) is "uncontrollable": see [28], Sect. 5.2. This means that the elements of $M_{c}^{*}$ are "free" (i.e. subject to no relation) whereas those of $M_{u}^{*}$ satisfy an autonomous differential equation and are completely determined by their initial conditions. The correspondence between the decompositions (13) and (17) is partly explained by Remark 7, since the module $\mathscr{C}^{\infty}(\Re ; \Re)$ is injective. It is further explained below in a slightly different context (see Proposition 18).

\section{Case (I)}

It is assumed in this section that $\boldsymbol{K}=\Re$ (case $(\mathrm{I})$ in $\$ 2.1 .1$ ). The endomorphism ring $\boldsymbol{E}$ of the $\boldsymbol{S}$-module $\tilde{\Delta}$ is identified with $\boldsymbol{S}$, according to Matlis' theory (see $\$ 2.1 .4$ ). 


\subsection{A key isomorphism}

\subsubsection{Continuous-time case}

Interconnecting two continuous-time systems from time 0 only, consists, from the analytic point of view, in multiplying a function, such as the function $e$ in the first row of (2), by $1-\Upsilon$, where $\Upsilon$ is the Heaviside function (i.e. $\Upsilon(t)=1$ for $t>0$ and 0 otherwise). Let $W=\mathscr{C}^{\infty}(\Re ; \Re)$ and set

$$
\Delta=\bigoplus_{\mu \geq 0} \Re \delta^{(\mu)}
$$

where $\delta$ is the Dirac distribution. The $\boldsymbol{R}$-module generated by $S_{0}:=(1-\Upsilon) W$ is (as $\Re$-vector space): $S=S_{0} \oplus \Delta$. The operator $\partial$ is an automorphism of the $\Re$-vector space $S$, and $\sigma=\partial^{-1}$ is the operator defined on $S$ by: $(\sigma w)(t)=\int_{+\infty}^{t} w(\varsigma) d \varsigma$. The space $S$ is an $\boldsymbol{L}$-vector space (and thus an $\boldsymbol{S}$-module which is an $\boldsymbol{R}$-module, by restriction of the ring of scalars), and $S_{0}$ is a $\boldsymbol{S}$-submodule of $S$. The $\boldsymbol{R}$-module $\Delta$ is not an $\boldsymbol{S}$-module, but $\Delta \cong \Re \frac{S}{S_{0}}:=\bar{\Delta} ; \bar{\Delta}$ is clearly an $\boldsymbol{L}$-vector space (and thus an $\boldsymbol{R}$ module which is an $\boldsymbol{S}$-module). The nature of the above isomorphism, denoted as $\tau$, can be further detailed:

Lemma 8. The isomorphism $\tau$, defined as: $\Delta \ni \lambda \delta \stackrel{\sim}{\rightarrow} \lambda \bar{\delta} \in \bar{\Delta}$, is $\boldsymbol{R}$-linear.

Proof. First, notice that any element of $\Delta$ (resp. $\bar{\Delta})$ can be uniquely expressed in the form $\lambda \delta$ (resp. $\lambda \bar{\delta}$ ) for some $\lambda \in \boldsymbol{R}$, thus $\tau$ is a well-defined $\mathbb{Z}$-isomorphism. In addition, for any $x \in \Delta$, such that $x=\lambda \delta, \lambda \in \boldsymbol{R}$, and any $\mu \in \boldsymbol{R}, \tau(\mu x)=\tau(\mu \lambda \delta)=$ $\mu \lambda \bar{\delta}=\mu \tau(x)$.

Therefore,

$$
\Delta \cong \boldsymbol{R} \frac{S}{S_{0}}:=\bar{\Delta}
$$

One has $\sigma \delta=\Upsilon-1$; setting $\bar{\delta}=\tau(\delta)$, one obtains $\sigma \bar{\delta}=0$, thus $\tilde{\delta}$ and $\bar{\delta}$ can be identified, as well as the $\boldsymbol{S}$-modules $\tilde{\Delta}$ and $\bar{\Delta}$. As a result, by (18), (11)

$$
\tilde{\Delta}=\bar{\Delta}=\bigoplus_{\mu \geq 0} \Re \tilde{\delta}^{(\mu)}
$$

In the remainder of this section, the canonical epimorphism $S \rightarrow \frac{S}{S_{0}}=\tilde{\Delta}$ is denoted as $\tilde{\phi}$. Let $\theta$ be the $\Re$-linear projection $S_{0} \oplus \Delta \rightarrow \Delta$; the following diagram is commutative:

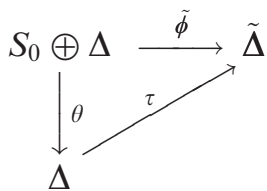




\subsubsection{Discrete-time case}

Let $\Upsilon$ be the sequence defined by $\Upsilon(t)=1$ for $t>0$ and 0 otherwise. Interconnecting a discrete-time system up to time 0 only, consists, from the analytic point of view, in multiplying a sequence, such as the sequence $e$ in (2), by $\Upsilon$. Let $W=\Re^{\mathbb{Z}}$ and $S_{0}=\Upsilon W$. Let $\Delta$ be defined as in (18), but where $\delta:=\partial \Upsilon$ is the "Kronecker sequence", such that $\delta(t)=1$ for $t=0$ and 0 otherwise (thus, $\Delta$ is the $\boldsymbol{R}$-module consisting of all sequences with left and finite support). The $\boldsymbol{R}$-module generated by $S_{0}$ is (as $\Re$-vector space) $S=S_{0} \oplus \Delta$. The operator $\partial$ is an automorphism of the $\Re$ vector space $S$, and $\sigma=\partial^{-1}$ is the operator defined on $S$ by: $(\sigma w)(t):=\sum_{j=-\infty}^{t-1} w(j)$; $S$ is an $\boldsymbol{L}$-vector space. The $\boldsymbol{R}$-isomorphism (19) still holds; the same identifications as in $\$ 3.1 .1$ can be made and the same notation can be used. Obviously, the discretetime case is completely analogous to the continuous-time one, and these two cases are no longer distinguished in the remainder of this section.

\subsection{Impulsive systems and behaviors}

\subsubsection{Impulsive and pseudo-impulsive behaviors}

Consider the temporal system (2), where $B(\partial) \in \boldsymbol{R}^{q \times k}$.

Proposition 9. The following properties are equivalent: (i) For any $e \in S_{0}^{q}$, there exists $w \in S^{k}$ such that (2) is satisfied. (ii) The matrix $B(\partial)$ is right regular, i.e. $q=r$, where $r$ is the rank of $B(\partial)$ over $\boldsymbol{R}$.

Proof. (i) $\Rightarrow$ (ii): If the matrix $B(\partial)$ is not right regular, $\bullet B(\partial)$ is not injective, i.e. there exists a nonzero element $\eta(\partial) \in \boldsymbol{R}^{q}$ (considered as a $1 \times q$ matrix with entries in $\boldsymbol{R})$ such that $\eta(\partial) B(\partial)=0$. Therefore, for $w \in S^{k}$ and $e \in S_{0}^{q}$ to satisfy (2), $e$ must satisfy the "compatibility condition" $\eta(\partial) e=0$. (ii) $\Rightarrow(\mathrm{i})$ : By (9), assuming that $q=r,(2)$ is equivalent to

$$
\left[\operatorname{diag}\left\{\sigma^{v_{i}}\right\}_{1 \leq i \leq r} \quad 0\right] v=h
$$

where $v=V(\sigma) w$ and $h=U(\sigma) e$; (22) is equivalent to $\sigma^{v_{i}} v_{i}=h_{i}, 1 \leq i \leq q$. For any $v_{i} \in \mathbb{Z}$ and any $h_{i} \in S_{0}, v_{i}=\partial^{v_{i}} h_{i}$ belongs to $S$. Therefore, (i) holds because $h$ spans $S_{0}^{q}$ as $e$ spans the same space (since $S_{0}$ is an $\boldsymbol{S}$-module).

Remark 10. The compatibility condition in the above proof is equivalent to the necessary and sufficient condition given in ([18], Theorem 5) for (2) to have solutions.

In the remainder of this section, the matrix $B(\partial) \in \boldsymbol{R}^{q \times k}$ is assumed to be right regular.

Notation 11. For any scalar operator $\omega$ and any integer $l \geq 1, \omega_{(l)}$ denotes the operator $\operatorname{diag}(\omega, \ldots, \omega)$, where $\omega$ is repeated $l$ times.

Definition 12. Let $\mathscr{W} \subset S^{k}$ be the space spanned by the elements $w$ satisfying (2) as $e$ spans $S_{0}^{q}$. The impulsive behavior of (2) is: $\mathscr{B}_{\infty}=\theta_{(k)} \mathscr{W}$. 
Definition 13. The pseudo-impulsive behavior of the temporal system (2) (or "associated with the matrix $\left.B(\partial)^{\prime \prime}\right)$ is: $\mathscr{A}_{\infty}=\tau_{(k)} \mathscr{B}_{\infty}$.

\subsubsection{Impulsive system}

Considering the Smith-MacMillan form at infinity (9) of $B(\partial)$ (with $q=r$ ), set $\Pi(\sigma)=\operatorname{diag}\left\{\sigma^{\bar{\pi}_{i}}\right\}_{1 \leq i \leq q}$ and $\Sigma(\sigma)=\operatorname{diag}\left\{\sigma^{\bar{\mu}_{i}}\right\}_{1 \leq i \leq q}$, so that $\operatorname{diag}\left\{\sigma^{v_{i}}\right\}_{1 \leq i \leq q}=$ $\Pi^{-1}(\sigma) \Sigma(\sigma)=\Sigma(\sigma) \Pi^{-1}(\sigma)$. By $(9), B(\partial)=A^{-1}(\sigma) B^{+}(\sigma)$, where $A(\sigma)=\Pi(\sigma) U(\sigma)$ and $B^{+}(\sigma)=\left[\begin{array}{ll}\Sigma(\sigma) & 0\end{array}\right] V(\sigma)$. The following result is classic (see, e.g., [37], Sect. 4.1, $(43))$ :

Lemma 14. (i) The above pair $\left(A(\sigma), B^{+}(\sigma)\right)$ is a left coprime factorization of $B(\partial)$ over S. (ii) Let $\left(A_{1}(\sigma), B_{1}^{+}(\sigma)\right)$ and $\left(A_{2}(\sigma), B_{2}^{+}(\sigma)\right)$ be two left coprime factorizations of $B(\partial)$ over $\boldsymbol{S}$; then, there exists a unimodular matrix $W(\sigma)$ over $\boldsymbol{S}$ such that $B_{2}^{+}(\sigma)=$ $W(\sigma) B_{1}^{+}(\sigma)$ and $A_{2}(\sigma)=W(\sigma) A_{1}(\sigma)$.

Let $\left(A(\sigma), B^{+}(\sigma)\right)$ be any left coprime factorization of $B(\partial)$ over $\boldsymbol{S}$. By Lemma 14, the module $M^{+}=$coker $\bullet B^{+}(\sigma)$ is uniquely defined from $B(\partial)$.

Definition 15. (i) The $\boldsymbol{S}$-module $M^{+}=\operatorname{coker} \bullet B^{+}(\sigma)$ is called the impulsive system associated with $B(\partial)$. (ii) The torsion submodule of $M^{+}$, written $\mathscr{T}\left(M^{+}\right)$, is called the module of uncontrollable poles at infinity of the temporal system (2) (or associated with the matrix $B(\partial))[5]^{8}$.

There exists a free module $\Phi^{+} \cong \boldsymbol{s} \boldsymbol{S}^{\kappa}, \kappa=k-q$, such that

$$
M^{+}=\Phi^{+} \oplus \mathscr{T}\left(M^{+}\right) .
$$

The ascending chain of invariant factors of $\mathscr{T}\left(M^{+}\right)$(possibly empty) is $\left(\sigma^{\mu_{\rho}}\right) \subset \cdots \subset\left(\sigma^{\mu_{1}}\right)$; thus $\mathscr{T}^{+}\left(M^{+}\right)$has the direct sum decomposition into cyclic indecomposable submodules:

$$
\mathscr{T}\left(M^{+}\right) \cong \boldsymbol{s} \bigoplus_{i=1}^{\rho} \tilde{C}_{\mu_{i}}
$$

The connection between the pseudo-impulsive behavior $\mathscr{A}_{\infty}$ and the impulsive system $M^{+}$is given by the following theorem, where $(.)^{*}:=\operatorname{Hom}_{\boldsymbol{S}}(., \tilde{\Delta})$ :

Theorem 16. $\mathscr{A}_{\infty}=\left(M^{+}\right)^{*}$.

Proof. By Definition 13 and the commutativity of the diagram (21), $\mathscr{A}_{\infty}$ is the $\boldsymbol{E}$ module (or the $\boldsymbol{S}$-module, since $\boldsymbol{E}$ and $\boldsymbol{S}$ are identified) consisting of all elements

\footnotetext{
8 It is called the module of input-decoupling zeros at infinity in the cited reference (in accordance with the terminology introduced in [4]), but this denomination should be reserved to the case where the inputs of the system have been chosen among its variables.
} 
$\tilde{w}=\tilde{\phi}_{(k)} w$ for which there exists $h \in S_{0}^{q}$ such that (22) is satisfied. With the notation in the proof of Proposition 9, this equation is equivalent to $\sigma^{v_{i}} v_{i}=h_{i}, 1 \leq i \leq q$. For any index $i$ such that $v_{i} \leq 0, v_{i}=\sigma^{-v_{i}} h_{i}$ belongs to $S_{0}$, thus $\tilde{v}_{i}=0$ (where $\tilde{v}_{i}:=\tilde{\phi} v_{i}$ ). Therefore, $\mathscr{A}_{\infty}$ is the $\boldsymbol{S}$-module consisting of all elements $\tilde{w}=V^{-1}(\sigma) \tilde{v}$ such that $\tilde{v} \in \tilde{\Delta}^{k}$ satisfies $\left[\begin{array}{ll}\Sigma(\sigma) & 0\end{array}\right] \tilde{v}=0$; as a result, $\mathscr{A}_{\infty}=\operatorname{ker} B^{+}(\sigma) \bullet$.

Remark 17. According to Theorem 16, the space $\mathscr{A}_{\infty}$ is a "behavior" in the sense specified in $\$ 2.2 .2$, i.e. a kernel, whereas the space $\mathscr{B}_{\infty}$ cannot be expressed in a so simple way (in this sense, there is an abuse of language in the expression "impulsive behavior"). This is why the notion of "pseudo-impulsive behavior" is very useful. The notion of "subbehavior" of a pseudo-impulsive behavior $\mathscr{A}_{\infty}$ is defined in accordance with the general definition in $\$ 2.2 .2$.

\subsubsection{Structure of impulsive behaviors}

The following result is analogous to the direct sum decomposition in $\$ 2.2 .2$.

Proposition 18. (i) There exist subbehaviors $\mathscr{A}_{\infty, c} \simeq\left(\Phi^{+}\right)^{*}$ and $\mathscr{A}_{\infty, u} \simeq\left(\mathscr{T}\left(M^{+}\right)\right)^{*}$ of $\mathscr{A}_{\infty}$ such that $\mathscr{A}_{\infty}=\mathscr{A}_{\infty, c} \oplus \mathscr{A}_{\infty, u}$. (ii) The subbehavior $\mathscr{A}_{\infty, c}$ satisfying this property is unique and such that $\mathscr{A}_{\infty, c} \cong \boldsymbol{s} \tilde{\Delta}^{\kappa}\left(\mathscr{A}_{\infty, c}\right.$ is called the "controllable pseudo-impulsive behavior"). (iii) $\mathscr{A}_{\infty, u} \cong \boldsymbol{s} \prod_{i=1}^{\rho} \tilde{C}_{\mu_{i}}$ (this subbehavior, unique up to $\boldsymbol{S}$-isomorphism, is said to be "uncontrollable").

Proof. (i): By (23), there exists a canonical $\mathbb{Z}$-isomorphism $j^{*}: \mathscr{A}_{\infty} \rightarrow\left(\Phi^{+}\right)^{*} \times$ $\left(\mathscr{T}\left(M^{+}\right)\right)^{*}$ given by $j^{*} \lambda=\left(\lambda j_{1}, \lambda j_{2}\right)$, where $j_{1}: \Phi^{+} \rightarrow M^{+}$and $j_{2}: \mathscr{T}\left(M^{+}\right) \rightarrow M^{+}$ are the canonical injections ([29], Theorem 2.4). Set $\mathscr{A}_{\infty, c}=j^{*-1}\left(\left(\Phi^{+}\right)^{*} \times 0\right)$ and $\mathscr{A}_{\infty, u}=j^{*-1}\left(0 \times\left(\mathscr{T}\left(M^{+}\right)\right)^{*}\right)$. Then, $\mathscr{A}_{\infty}=\mathscr{A}_{\infty, c} \oplus \mathscr{A}_{\infty, u}$ and by Lemma 5(i), $\mathscr{A}_{\infty, c} \simeq\left(\Phi^{+}\right)^{*}$ and $\mathscr{A}_{\infty, u} \simeq\left(\mathscr{T}\left(M^{+}\right)\right)^{*}$. (ii): As $\boldsymbol{E}=\boldsymbol{S}, \mathscr{A}_{\infty, c} \cong \boldsymbol{s}\left(\Phi^{+}\right)^{*} \cong \boldsymbol{s} \tilde{\Delta}^{\kappa}$. By Lemma 5(iii), there exists a natural integer $\mu$ such that $\sigma^{\mu} \mathscr{A}_{\infty, u}=0$, thus $\sigma^{\mu} \mathscr{A}_{\infty}=$ $\sigma^{\mu} \mathscr{A}_{\infty, c}$. Assuming that there exist two other $\boldsymbol{S}$-modules $\mathscr{A}_{\infty, c}^{\prime} \simeq\left(\Phi^{+}\right)^{*}$ and $\mathscr{A}_{\infty, u}^{\prime} \simeq$ $\left(\mathscr{T}\left(M^{+}\right)\right)^{*}$ such that $\mathscr{A}_{\infty}=\mathscr{A}_{\infty, u}^{\prime} \oplus \mathscr{A}_{\infty, c}^{\prime}$, one obtains $\sigma^{\mu} \mathscr{A}_{\infty, c}=\sigma^{\mu} \mathscr{A}_{\infty, c}^{\prime}$, thus $\mathscr{A}_{\infty, c}=\mathscr{A}_{\infty, c}^{\prime}$ by Lemma 5(iv), since $\tilde{\Delta}$ is a cogenerator (see $\S 2.1 .4$ ). (iii): $\mathscr{A}_{\infty, u} \cong \boldsymbol{s} \mathscr{T}\left(\left(M^{+}\right)\right)^{*} \cong \boldsymbol{s} \prod_{i=1}^{\rho} \tilde{C}_{\mu_{i}}^{*}$ by $(24)$ and $\tilde{C}_{\mu_{i}}^{*}=\tilde{C}_{\mu_{i}}$, according to Matlis' theory.

For any integer $\mu \geq 1$, set

$$
C_{\mu}=\tau^{-1}\left(\tilde{C}_{\mu}\right)=\bigoplus_{i=1}^{\mu} \Re \delta^{(i-1)} .
$$

The following theorem is an obvious consequence of Proposition 18:

Theorem 19. Let us consider the temporal system with matrix $B(\partial)$ (assumed to be right regular). Its impulsive behavior $\mathscr{B}_{\infty}$ can be expressed as: $\mathscr{B}_{\infty}=\mathscr{B}_{\infty, c} \oplus \mathscr{B}_{\infty, u}$, 
where $\mathscr{B}_{\infty, c}:=\tau_{(k)}^{-1} \mathscr{A}_{\infty, c} \cong \Re \Delta^{\kappa}$ and $\mathscr{B}_{\infty, u}=\tau_{(k)}^{-1} \mathscr{A}_{\infty, u} \cong \Re \prod_{i=1}^{\rho} C_{\mu_{i}}$ (the space $\mathscr{B}_{\infty, c}$, which is uniquely defined, is called the "controllable impulsive behavior", and the impulsive behavior $\mathscr{B}_{\infty, u}$, unique up to $\Re$-isomorphism, is said to be "uncontrollable").

\subsubsection{Temporal interconnections}

More details about temporal interconnections can now be given. Consider two temporal systems

$$
\left\{\begin{array}{l}
B_{i}(\partial) w^{i}(t)=e^{i}(t), \quad t \in \mathbb{T} \\
e^{i}(t)=0, \quad t \in \mathbb{T}_{0}
\end{array}\right.
$$

$(i=1,2)$. They can be interconnected; the matrices $J_{1}$ and $J_{2}$ in (15) are assumed to have their coefficients in $\Re$. According to Sect. 1 and $\$ 2.2 .1$, we are led to the following definition:

Definition 20. The interconnected temporal system is defined by

$$
\left\{\begin{array}{l}
B(\partial) w(t)=e(t), \quad t \in \mathbb{T} \\
e(t)=0, \quad t \in \mathbb{T}_{0}
\end{array}\right.
$$

where the matrix $B(\partial):=\left[\begin{array}{cc}B_{1}(\partial) & 0 \\ 0 & B_{2}(\partial) \\ J_{1} & -J_{2}\end{array}\right]$ is assumed to be right regular; $J_{1}$ and $J_{2}$ are called the interconnection matrices.

One has the following result:

Theorem 21. The impulsive system $M^{+}$of the interconnected temporal system is defined as

$$
M^{+}=\operatorname{coker} \bullet\left[\begin{array}{cc}
B_{1}^{+}(\sigma) & 0 \\
0 & B_{2}^{+}(\sigma) \\
J_{1} & -J_{2}
\end{array}\right]
$$

where $\left(A_{i}(\sigma), B_{i}^{+}(\sigma)\right)$ is any left coprime factorization over $\boldsymbol{S}$ of $B_{i}(\partial)(i=1,2)$.

Proof. Set

$$
B^{+}(\sigma)=\left[\begin{array}{cc}
B_{1}^{+}(\sigma) & 0 \\
0 & B_{2}^{+}(\sigma) \\
J_{1} & -J_{2}
\end{array}\right], \quad A(\sigma)=\left[\begin{array}{ccc}
A_{1}(\sigma) & 0 & 0 \\
0 & A_{2}(\sigma) & 0 \\
0 & 0 & I_{p}
\end{array}\right]
$$


where $p$ is the number of rows of the matrices $J_{1}$ and $J_{2}$. Obviously, $\left(A(\sigma), B^{+}(\sigma)\right)$ is a left coprime factorization over $\boldsymbol{S}$ of $B(\partial)$; therefore, the proposition is proved, according to Definition 15.

Remark 22. By Theorem 21 and (15), $M^{+}$can be written as a fibered sum of the impulsive systems $M_{i}^{+}=$coker $\bullet B_{i}^{+}$. In other words, the impulsive system of the interconnected temporal system is obtained by interconnecting the impulsive systems of the temporal subsystems.

\section{Case (II)}

It is assumed in this section that the "coefficient ring" $\boldsymbol{K}$ is a field (case (II) in §2.1.1). As said in Section 1, assuming that the coefficients are time-varying but belong to a field amounts to discarding singularities. Nevertheless, there is no natural definition of the impulsive behavior of a temporal system in the present case and the key isomorphism $\tau$ in Lemma 8 is no longer valid. The results in this section are essentially formal and may be viewed as an introduction to those in Section 5.

Let $B(\partial) \in \boldsymbol{R}^{q \times k}$ be a right regular matrix. According to $\$ \S 2.1 .1,2.1 .3$, the statement in Lemma 14 remains valid. Therefore, the following definitions make sense (the first one was already given in [5]):

Definition 23. The impulsive system and the module of uncontrollable poles at infinity associated with the matrix $B(\partial)$ are defined as in Definition 15 , i.e. as $M^{+}$and $\mathscr{T}\left(M^{+}\right)$, respectively.

Definition 24. The pseudo-impulsive behavior $\mathscr{A}_{\infty}$ associated with $B(\partial)$ is defined as: $\mathscr{A}_{\infty}=\left(M^{+}\right)^{*}$, where $(.)^{*}:=\operatorname{Hom}_{\boldsymbol{S}}(., \tilde{\Delta})$.

Matlis' theory does no longer apply in the present context, thus the ring $\boldsymbol{S}$ and the endomorphism ring $\boldsymbol{E}$ of the $\boldsymbol{S}$-module $\tilde{\Delta}$ must be distinguished. However:

Proposition 25. For any natural integer $\mu, \tilde{C}_{\mu}^{*}=\tilde{C}_{\mu}$.

Proof. For $\mu=0, \tilde{C}_{\mu}=\tilde{C}_{\mu}^{*}=0$. For $\mu \geq 1, \tilde{C}_{\mu}^{*}$ is the set of all elements $x \in \tilde{\Delta}$ such that $\sigma^{\mu} x=0$. Obviously, $\tilde{\delta}^{(i-1)}$ belongs to $\tilde{C}_{\mu}^{*}$ if, and only if $1 \leq i \leq \mu$. By (10), $\tilde{C}_{\mu}^{*} \subset \tilde{C}_{\mu}$. Let us prove by induction the reverse inclusion. By $\tilde{\tilde{C}}_{\tilde{\tilde{\delta}}}(5)$, for any $a \in \boldsymbol{K}$, $\sigma a \tilde{\delta}=\left(a^{\beta} \sigma-\sigma a^{\beta \gamma} \sigma\right) \tilde{\delta}=0$, which implies that $\tilde{C}_{1}=\boldsymbol{K} \tilde{\delta} \subset \tilde{C}_{1}^{*}$. Assuming that $\tilde{C}_{\mu} \subset \tilde{C}_{\mu}^{*}, \mu \geq 1$, let $a \in \boldsymbol{K}$; then, $\sigma^{\mu+1} a \tilde{\delta}^{(\mu)}=\sigma^{\mu}\left(a^{\beta}-\sigma a^{\beta \gamma}\right) \tilde{\delta}^{(\mu-1)}$; by hypothesis, $a^{\beta} \tilde{\delta}^{(\mu-1)}$ and $\sigma a^{\beta \gamma} \tilde{\delta}^{(\mu-1)}$ belong to $\tilde{C}_{\mu}^{*}$, thus $\sigma^{\mu+1} a \tilde{\delta}^{(\mu)}=0$, which implies that $\tilde{C}_{\mu+1} \subset \tilde{C}_{\mu+1}^{*}$.

The direct sum decompositions (23) and (24) are correct [5] and $\tilde{\Delta}$ is a cogenerator of $\boldsymbol{s} \mathscr{M}$. Therefore, by Proposition 25, one has the following result, in place of the statement of Proposition 18: 
Proposition 26. (i) There exist subbehaviors $\mathscr{A}_{\infty, c} \simeq\left(\Phi^{+}\right)^{*}$ and $\mathscr{A}_{\infty, u} \simeq\left(\mathscr{T}\left(M^{+}\right)\right)^{*}$ of $\mathscr{A}_{\infty}$ such that $\mathscr{A}_{\infty}=\mathscr{A}_{\infty, c} \oplus \mathscr{A}_{\infty, u}$. (ii) The subbehavior $\mathscr{A}_{\infty, c}$ satisfying this property is unique and such that $\mathscr{A}_{\infty, c} \cong_{\boldsymbol{E}} \tilde{\Delta}^{\kappa}$ ( $\mathscr{A}_{\infty, c}$ is called the "controllable pseudo-impulsive behavior”'). (iii) $\mathscr{A}_{\infty, u} \cong_{\boldsymbol{E}} \prod_{i=1}^{\rho} \tilde{C}_{\mu_{i}}$ (this subbehavior, unique up to $\boldsymbol{E}$-isomorphism, is said to be "uncontrollable").

\section{Case (III)}

\subsection{Impulsive behavior of a temporal system with time-varying coefficients}

The key isomorphism valid again. Case (III) in $\$ 2.1 .1$ is now considered. In the case of continuous-time temporal systems, let us slightly modify the definition of the spaces $W, S_{0}$ and $S$ in $\S 3.1 .1$ (in the case of discrete-time temporal systems, their definition, as given in $\$ 3.1 .2$, is left unchanged):

For any integer $n \geq 1$, let $W_{n}=\mathscr{C}^{\infty}\left(I_{n} ; \Re\right)$, where $\left.I_{n}=\right]-\frac{1}{n},+\infty\left[\right.$. As $I_{n+1} \subset I_{n}$, $W:=\lim W_{n}$ is the space of germs of $\mathscr{C}^{\infty}$ functions on an open connected neighborhood of $\left[0,+\infty\right.$ [. Let $S_{0}=(1-\Upsilon) W$ and $S=S_{0} \oplus \Delta$, where $\Delta$ is defined by (18). The space $S$ is still the $\boldsymbol{R}$-module generated by $S_{0}$, and the "continuous time derivative" $\partial$ is an automorphism of the $\Re$-vector space $S$. For any $w \in S$, there exists $n \geq 1$ such that $w \in(1-\Upsilon) W_{n} \oplus \Delta$, thus $(\sigma w)(t):=\int_{+\infty}^{t} w(\varsigma) d \varsigma$ is defined for any $t \in I_{n} ; \sigma$ is an automorphism of $S$, and $\sigma=\partial^{-1}$.

As in Section 3, in both the continuous- and discrete-time cases, $S_{0}$ and $S$ are $\boldsymbol{S}$-modules and $S$ is an $\boldsymbol{L}$-module (a property which was lost in Section 4). The canonical $\boldsymbol{S}$-linear epimorphism $S \rightarrow \frac{S}{S_{0}}:=\bar{\Delta}$ is denoted as $\bar{\phi}$.

Proposition 27. (i) For any natural integer $\mu$, the $\Re$-vector space $C_{\mu}:=\bigoplus_{i=1}^{\mu} \boldsymbol{K} \delta^{(i-1)}$ satisfies the following equality: $C_{\mu}=\bigoplus_{i=1}^{\mu} \Re \delta^{(i-1)}$. (ii) The $\Re$-vector space $\Delta$ defined by (18) has a natural structure of $\boldsymbol{R}$-module and $\Delta=\bigoplus_{\mu \geq 0} \boldsymbol{K} \delta^{(\mu)}$. (iii) The quotient $\bar{\Delta}$ is an $\mathbf{L}$-module (and thus an $\boldsymbol{R}$-module which is an $\boldsymbol{S}$-module, by restriction of the ring of scalars), and it satisfies the following equality: $\bar{\Delta}=\bigoplus_{\mu \geq 0} \boldsymbol{K} \bar{\delta}^{(\mu)}=\bigoplus_{\mu \geq 0} \Re \bar{\delta}^{(\mu)}$. (iv) The $\boldsymbol{R}$-isomorphism $\tau$ defined as in Lemma 8 and (19) still holds and, setting $\bar{C}_{\mu}=\tau\left(C_{\mu}\right)$, one has $\bar{C}_{\mu}=\bigoplus_{i=1}^{\mu} \boldsymbol{K} \bar{\delta}^{(i-1)}=\bigoplus_{i=1}^{\mu} \Re \bar{\delta}^{(i-1)}$.

Proof. (i): Obviously, $\bigoplus_{i=1}^{\mu} \Re \delta^{(i-1)} \subset \bigoplus_{i=1}^{\mu} \boldsymbol{K} \delta^{(i-1)}$. Let us prove the converse by induction. Let $a \in \boldsymbol{K}$; as $a \delta=a(0) \delta$ (in both the continuous- and discrete-time cases), $\boldsymbol{K} \delta=\Re \delta$. Assuming that $\bigoplus_{i=1}^{\mu} \boldsymbol{K} \delta^{(i-1)} \subset \bigoplus_{i=1}^{\mu} \Re \delta^{(i-1)}$, let $a \in \boldsymbol{K}$. One has $a \delta^{(\mu)}=$ $a \partial \delta^{(\mu-1)}$; setting $a=b^{\alpha}$, one obtains $a \delta^{(\mu)}=\left(\partial b-b^{\gamma}\right) \delta^{(\mu-1)}$ by (4), and by hypothesis $b \delta^{(\mu-1)} \in \bigoplus_{i=1}^{\mu} \Re \delta^{(i-1)}$, thus $\partial b \delta^{(\mu-1)} \in \bigoplus_{i=1}^{\mu+1} \Re \delta^{(i-1)}$. Finally, $a \delta^{(\mu)} \in \bigoplus_{i=1}^{\mu+1} \Re \delta^{(i-1)}$, thus $\bigoplus_{i=1}^{\mu+1} \boldsymbol{K} \delta^{(i-1)} \subset \bigoplus_{i=1}^{\mu+1} \Re \delta^{(i-1)}$. (ii) is an obvious consequence of (i), since $\Delta=\lim C_{\mu}$. (iii) and (iv) are then clear, by the same rationale as in the proof of Lemma 8 .

By Proposition 27, the diagram below (where the $\Re$-linear projection $\theta$ is defined as in $\S 3.1 .1)$ is commutative and, in the present section, must be considered in place of (21): 


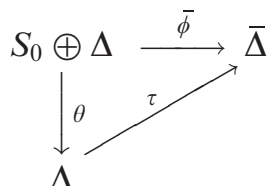

Difficulties. The structure of the ring $\boldsymbol{S}$ is not as simple as in Section 4, since $\boldsymbol{S}$ is no longer a principal ideal domain. In addition, an important difference with the situation in Section 3 appears in the following proposition:

Proposition 28. Let $\psi: \tilde{\Delta} \rightarrow \bar{\Delta}$ be defined as: $\psi(\lambda \tilde{\delta})=\lambda \bar{\delta}$, where $\lambda \in \boldsymbol{R}$. (i) $\psi$ is an S-epimorphism. (ii) $0 \neq t \tilde{C}_{1} \subset \operatorname{ker} \psi$.

Proof. (i): Any element of $\tilde{\Delta}$ can be uniquely expressed in the form $\lambda \tilde{\delta}, \lambda \in \boldsymbol{R}$, and every element of $\bar{\Delta}$ can be expressed in the form $\lambda \bar{\delta}$ for some $\lambda \in \boldsymbol{R}$, thus $\psi: \tilde{\Delta} \rightarrow \bar{\Delta}$ is a well-defined $\mathbb{Z}$-epimorphism. Let us show that $\psi$ is $\boldsymbol{S}$-linear. Let $x=\lambda \tilde{\delta} \in \tilde{\Delta}$, and (as a result of an Euclidean division by $\partial$ ) write $\lambda=\lambda_{0}+\partial \lambda_{1}$, where $\lambda_{0} \in \boldsymbol{K}$ and $\lambda_{1} \in \boldsymbol{R}$; then, $\sigma x=\lambda_{1} \tilde{\delta}$ (since $\sigma$ is a left inverse of $\partial$ : see $\S 2.1 .4$ ). Therefore, $\psi(\sigma x)=$ $\lambda_{1} \bar{\delta}=\sigma \psi(x)$. (ii): As $t \in \boldsymbol{K}$ and $\tilde{C}_{1} \cong \boldsymbol{K} \boldsymbol{K}, t \tilde{C}_{1}$ is nonzero. As already said in the proof of Proposition 27(i), $a \delta=a(0) \delta$, thus $t \bar{\delta}=0$, therefore $\psi\left(t \tilde{C}_{1}\right)=0$ since $\tilde{C}_{1}=\boldsymbol{K} \tilde{\delta}$.

By Proposition $28(\mathrm{i}), \bar{\Delta} \cong \boldsymbol{s} \tilde{\Delta} / \operatorname{ker} \psi$, and by Proposition $28(\mathrm{ii})$, $\operatorname{ker} \psi$ is nonzero. Therefore, $\bar{\Delta}$ and $\tilde{\Delta}$ cannot be identified, and (20) is no longer true.

\section{Impulsive behavior of a semiregular temporal system}

Definition 29. (i) The temporal system (2) is said to be semiregular if, for any $e \in S_{0}^{q}$, there exists $w \in S^{k}$ such that (2) holds. (ii) The impulsive behavior $\mathscr{B}_{\infty}$ of a semiregular temporal system is defined as in Definition 12.

For the temporal system (2) to be semiregular, $B(\partial)$ must be right regular, as shown by the proof of Proposition 9. Let us further study semiregularity, assuming that $\boldsymbol{K}=\Re[t]$. Let $B(\partial) \in \boldsymbol{R}^{q \times k}$ be the matrix of the temporal system (2). According to Lemma 1, there exist two unimodular matrices $\bar{U}(t, \sigma)$ and $\bar{V}(t, \sigma)$ over $\mathbf{L}=$ $\Re((\sigma))[t ; 1, \varepsilon]$ and a nonzero element $\varpi(t, \sigma) \in \boldsymbol{L}$ such that

$$
\bar{U}(t, \sigma) B(\partial) \bar{V}^{-1}(t, \sigma)=\left[\begin{array}{cc}
\operatorname{diag}\{1, \ldots, 1, \varpi(t, \sigma)\} & 0 \\
0 & 0
\end{array}\right] .
$$

The matrix in the right-hand side of the above equality is the Smith form of $B(\partial)$ over $\boldsymbol{L}([7], \S 8.1$, Corollary 1.2).

Theorem 30. Assuming that $\boldsymbol{K}=\Re[t]$, the temporal system (2) is semiregular if, and only if its matrix $B(\partial)$ is right regular and $\bar{U}_{q}(t, \sigma) S_{0}^{q} \subset \varpi(t, \sigma) S$, where $\bar{U}_{q}(t, \sigma)$ is the last row of $\bar{U}(t, \sigma)$. 
Proof. Assuming that $B(\partial)$ is right regular, (2) is equivalent to $\operatorname{diag}\{1, \ldots, 1, \varpi(t, \sigma)\} \bar{v}$ $=\bar{U}(t, \sigma) e$, where $\bar{v}$ is the vector formed from the $q$ first rows of $\bar{V}(t, \sigma) w$. The only problem is with the last component $\bar{v}_{q}$ of $\bar{v}$. The equation $\varpi(t, \sigma) \bar{v}_{q}=\bar{U}_{q}(t, \sigma) e$ has a solution $\bar{v}_{q} \in S$ for any $e \in S_{0}^{q}$, if, and only if $\bar{U}_{q}(t, \sigma) S_{0}^{q} \subset \varpi(t, \sigma) S$.

Corollary 31. In the continuous-time case with $\boldsymbol{K}=\Re[t]$, let $B(\partial) \in \boldsymbol{R}^{q \times k}$ be the matrix of a temporal system, and assume that $B(\partial)$ is right regular. If the coefficient of least order of $\varpi(t, \sigma)$ (where $\varpi(t, \sigma)$ is considered as an element of $\boldsymbol{K}\left(\left(\sigma ; 1, \frac{d}{d t}\right)\right)$ ) does not vanish at $t=0$, then the temporal system is semiregular.

Proof. The element $\varpi(t, \sigma)$ can be written as: $\varpi(t, \sigma)=\sum_{i \geq N} f_{i}(t) \sigma^{i}$, where $N \in \mathbb{Z}$, every $f_{i}(t)$ belongs to $\boldsymbol{K}=\Re[t]$ and $f_{N}(t) \neq 0 ; f_{N}(t)$ is the coefficient of least order of $\varpi(t, \sigma)$. Let us assume that $f_{N}(0) \neq 0$, and set $\varpi(t, \sigma)=f_{N}(t) u(t, \sigma) \sigma^{N}$, where $u(t, \sigma)=1-g(t, \sigma)$ and $g(t, \sigma)=-\sum_{i \geq N+1}\left(f_{i}(t) / f_{N}(t)\right) \sigma^{i-N}$. The elements $u(t, \sigma)$ and $f_{N}(t)$ are units of the ring $W\left[\left[\sigma ; 1, \frac{d}{d t}\right]\right]$ (the former with inverse $\left.1+\sum_{j \geq 0}(g(t, \sigma))^{j}\right)$. In addition, $\sigma^{N} S=S$. Finally, as $S$ is a $W\left[\left[\sigma ; 1, \frac{d}{d t}\right]\right]$-module, $\varpi(t, \sigma) S=S$, and $\bar{U}_{q}(t, \sigma) S_{0}^{q} \subset S$.

Example 32. Let us consider the temporal system with matrix

$$
B(\partial)=\left[\begin{array}{ccc}
\partial+t & t & 0 \\
0 & 0 & 1 \\
1 & 0 & 1
\end{array}\right] .
$$

The Smith form of $B(\partial)$ over $\boldsymbol{L}$ is $\operatorname{diag}(1,1, t)$, and $\bar{U}_{3}(t, \sigma)=\left[\begin{array}{lll}1 & \partial & -\partial\end{array}\right]$; thus, $\bar{U}_{3}(t, \sigma) S_{0}^{3}=S_{0} \oplus \Re \delta$. As $S_{0} \oplus \Re \delta$ is not included in $t S$, this temporal system is nonsemiregular.

\subsection{Regular temporal systems}

\subsubsection{Definition and properties of regularity}

Definition 33. The temporal system (2) is said to be regular if the matrix $B(\partial) \in \boldsymbol{R}^{q \times k}$ is right regular and has a Smith-MacMillan form at infinity, i.e. if there exist unimodular matrices $U(\sigma) \in \boldsymbol{S}^{q \times q}, V(\sigma) \in \boldsymbol{S}^{k \times k}$, as well as integers $v_{1}, \ldots, v_{r}, v_{1} \leq \cdots \leq v_{r}$, such that (9) holds with $r=q$. The structural indexes of the zeros at infinity of such a matrix $B(\partial)$ are the integers $\mu_{i}(1 \leq i \leq \rho)$ as defined in Section 2.1.3.

The following result is clear (by the same rationale as in the proof of Proposition 9):

Proposition 34. A regular temporal system is semiregular.

Definition 35. $\boldsymbol{s} \mathscr{M}^{\text {struc }}$ is the full subcategory of $\boldsymbol{s} \mathscr{M}^{f}$ whose objects are the modules $M^{+}$of the form (23), where $\mathscr{T}\left(M^{+}\right)$is zero or is such that there exist natural integers $\rho$ and $\mu_{i}, 1 \leq i \leq \rho, 1 \leq \mu_{1} \leq \cdots \leq \mu_{\rho}$, for which (24) holds. 
Proposition 36. Let $B(\partial) \in \boldsymbol{R}^{q \times k}$ be the matrix of definition of a regular temporal system. (i) The matrix $B(\partial)$ has a CLCF $\left(A(\sigma), B^{+}(\sigma)\right)$ over $\boldsymbol{S}$. (ii) Let $\left(A(\sigma), B^{+}(\sigma)\right)$ be any $C L C F$ of $B(\partial)$ over $\boldsymbol{S} ; M^{+}:=$coker $\bullet B^{+}(\sigma)$ is uniquely defined from $B(\partial)$ and is an object of $\boldsymbol{s} \mathscr{M}^{\text {struc }}$.

Proof. (i): Let $\Pi(\sigma)$ and $\Sigma(\sigma)$ be the matrices as defined in the beginning of $\$ 3.2 .2$. The pair $(\Pi(\sigma),[\Sigma(\sigma) \quad 0])$ is completely left coprime over $\Re[[\sigma]] \subset \boldsymbol{S}$. Thus, there exist matrices $X(\sigma), Y(\sigma)$ over $\Re[[\sigma]]$ such that the following matrix $Q(\sigma)$ is unimodular over $\Re[[\sigma]]$ :

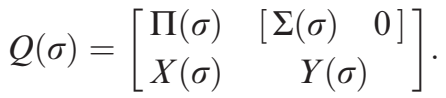

With the notation in the beginning of $\$ 3.2 .2, B(\partial)=A^{-1}(\sigma) B^{+}(\sigma)$, where $A(\sigma)=$ $\Pi(\sigma) U(\sigma), B^{+}(\sigma)=\left[\begin{array}{ll}\Sigma(\sigma) & 0\end{array}\right] V(\sigma)$, and where the matrices $U(\sigma)$ and $V(\sigma)$ are unimodular over $\boldsymbol{S}$. Now,

$$
Q(\sigma)\left[\begin{array}{cc}
U(\sigma) & 0 \\
0 & V(\sigma)
\end{array}\right]=\left[\begin{array}{cc}
A(\sigma) & B^{+}(\sigma) \\
* & *
\end{array}\right]
$$

(where each " $*$ " denotes a non specified submatrix). As the matrix in the left-hand side of the above equality is unimodular over $\boldsymbol{S},\left(A(\sigma), B^{+}(\sigma)\right)$ is a CLCF of $B(\partial)$ over $\boldsymbol{S}$. (ii): For the above CLCF, $\operatorname{coker} \bullet B^{+}(\sigma) \cong \boldsymbol{s} \operatorname{coker} \bullet[\Sigma(\sigma) \quad 0]$; in addition, statement (ii) of Lemma 14 is still correct (by the same rationale as in, e.g., [37], Sect. $4.1,(43))$.

\subsubsection{Impulsive modules and behaviors of regular temporal systems}

Proposition 37. The S-module $\tilde{\Delta}$ is a cogenerator for the subcategory $\boldsymbol{s} \mathscr{M}^{\text {struc }}$.

Proof. 1) This statement means that for every nonzero $f: M^{+} \rightarrow N^{+}, M^{+} \in \boldsymbol{s} \mathscr{M}^{\text {struc }}$, $N^{+} \in \boldsymbol{S} \mathscr{M}^{\text {struc }}$, there exists $g: N^{+} \rightarrow \tilde{\Delta}$ such that $g f \neq 0$. As $\boldsymbol{S} \in \boldsymbol{S} \mathscr{M}^{\text {struc }}$, this property is equivalent to the following one: for any $N^{+} \in \boldsymbol{S} \mathscr{M}^{\text {struc }}$ and any $0 \neq x \in N^{+}$, there exists an $\boldsymbol{S}$-morphism $h: N^{+} \rightarrow \tilde{\Delta}$ such that $h(x) \neq 0$ (see [19], Proposition (19.6) and the proof of this proposition $)^{9}$. 2) If $N^{+}=\tilde{C}_{\mu}, \mu \geq 1$, and $0 \neq x \in N^{+}$, $\lambda_{\mu}(x)=x \neq 0$, where $\lambda_{\mu}: \tilde{C}_{\mu} \rightarrow \tilde{\Delta}$ is the canonical injection. 3) Let us prove that for any $0 \neq x \in \boldsymbol{S}$, there exists an $\boldsymbol{S}$-morphism $f: \boldsymbol{S} \rightarrow \tilde{\Delta}$ such that $f(x) \neq 0$. Let $m$ be any natural integer; the morphism $\tilde{f}_{m}: \boldsymbol{S} \rightarrow \tilde{\Delta}$, defined as $\tilde{f}_{m}(y)=y \tilde{\boldsymbol{\delta}}^{(m)}$, is $\boldsymbol{S}$-linear. Writing $y=\sum_{i \geq 0} y_{i} \sigma^{i}, y_{i} \in \boldsymbol{K}$, one obtains $\tilde{f}_{m}(y)=\sum_{i \geq 0} y_{i} \tilde{\delta}^{(m-i)}$. Since $0 \neq x=$ $\sum_{i \geq 0} x_{i} \sigma^{i}$, there exists a nonzero $x_{j} \in \boldsymbol{K}$, thus $\tilde{f}_{j}(x) \neq 0$, and 3) is proved. 4) As any $N^{+} \in \boldsymbol{S} \mathscr{M}^{\text {struc }}$ is a direct sum of cyclic modules, by 2) and 3), for any $N^{+} \in \boldsymbol{S} \mathscr{M}^{\text {struc }}$ and any $0 \neq x \in N^{+}$, there exists an $\boldsymbol{S}$-morphism $h: N^{+} \rightarrow \tilde{\Delta}$ such that $h(x) \neq 0$.

\footnotetext{
${ }^{9}$ Notice that $\tilde{\Delta} \notin_{\boldsymbol{S}} \mathscr{M}^{\text {struc }}$. Similarly, one can prove that the module $\tilde{\Delta}$ is injective for the subcategory $\mathscr{M}^{\text {struc }}$.
} 
By Proposition 36, the following definition is relevant:

Definition 38. The impulsive system and the module of uncontrollable poles at infinity of a regular temporal system are defined as in Definition 15, i.e. as $M^{+}$and $\mathscr{T}\left(M^{+}\right)$, respectively. Its pseudo-impulsive behavior $\mathscr{A}_{\infty}$ is defined as in Definition 24 .

Proposition 39. For a regular temporal system, the statements of Propositions 25 and 26 are valid.

Proof. The proofs of the above propositions are still valid, using Proposition 37 for the latter.

The structure of the impulsive behavior of a regular temporal system is now completely elucidated:

Theorem 40. Let us consider a regular temporal system with matrix $B(\partial) \in \boldsymbol{R}^{q \times k}$. Let $\mathscr{A}_{\infty}$ and $\mathscr{A}_{\infty, c}$ be its pseudo-impulsive behavior and its controllable pseudo-impulsive behavior, respectively, and let $\mathscr{A}_{\infty, u}$ be an uncontrollable pseudo-impulsive behavior such as in Proposition 26. (i) The impulsive behavior $\mathscr{B}_{\infty}$ of the temporal system is expressed as: $\mathscr{B}_{\infty}=\tau_{(k)}^{-1} \psi_{(k)} \mathscr{A}_{\infty}$ (where $\psi$ is as defined in Proposition 28). (ii) The following decomposition holds: $\mathscr{B}_{\infty}=\mathscr{B}_{\infty, c} \oplus \mathscr{B}_{\infty, u}$, where $\mathscr{B}_{\infty, c}:=\tau_{(k)}^{-1} \psi_{(k)} \mathscr{A}_{\infty, c} \cong_{\Re} \Delta^{k}$ and $\mathscr{B}_{\infty, u}=\tau_{(k)}^{-1} \psi_{(k)} \mathscr{A}_{\infty, u} \cong \Re \prod_{i=1}^{\rho} C_{\mu_{i}}$ (the space $\mathscr{B}_{\infty, c}$, which is uniquely defined, is called the "controllable impulsive behavior" and the impulsive behavior $\mathscr{B}_{\infty, u}$, unique up to $\Re$-isomorphism, is said to be "uncontrollable").

Proof. (i): By the commutative diagram (27), $\tau_{(k)} \mathscr{B}_{\infty}$ is the set of all elements $\bar{w}=\bar{\phi}_{(k)} w$ such that $B^{+}(\sigma) \bar{w}=0$ (as shown by the proof of Theorem 16), thus $\tau_{(k)} \mathscr{B}_{\infty}=\psi_{(k)} \mathscr{A}_{\infty}$. (ii) is an obvious consequence of Proposition 39.

Regarding interconnected temporal systems, Definition 20 is relevant. However:

Lemma 41. The temporal system resulting from the temporal interconnection of two regular temporal systems can be non-semiregular.

Proof. Consider the temporal systems defined by $B_{1}(\partial)=\left[\begin{array}{ll}\partial+t & t\end{array}\right]$ and $B_{2}(\partial)=1$, which are regular, and assume that the interconnection matrices are $J_{1}=\left[\begin{array}{ll}1 & 0\end{array}\right]$ and $J_{2}=1$. The resulting temporal system, which is the one in Example 32, is nonsemiregular.

Therefore, the statement of Theorem 21 must be modified as follows:

Theorem 42. Consider two regular temporal systems, interconnected as in Definition 20. Assuming that the interconnected temporal system is regular, its impulsive system $\mathrm{M}^{+}$ is given by (26), where $\left(A_{i}(\sigma), B_{i}^{+}(\sigma)\right)$ is any CLCF over $\boldsymbol{S}$ of $B_{i}(\partial)(i=1,2)$. 


\subsection{Examples}

\subsubsection{A regular example}

Consider the following example (in the continuous- or discrete-time case):

$$
B(\partial)=\left[\begin{array}{cccc}
-1 & \partial^{2}+t & 0 & 0 \\
0 & 0 & \partial^{2} & -1 \\
0 & 1 & -1 & 0
\end{array}\right]
$$

and write $w=\left[\begin{array}{llll}u_{1} & y_{1} & u_{2} & y_{2}\end{array}\right]^{T}$. This system can be viewed as the series interconnection of System 1, with input $u_{1}$, output $y_{1}$ and equation $\ddot{y}_{1}+t y_{1}-u_{1}=0$, with System 2 with input $u_{2}$, output $y_{2}$ and equation $\ddot{u}_{2}-y_{2}=0$; the interconnection equation is $u_{2}=y_{1}$. Assuming that the interconnection is temporal, in the resulting interconnected temporal system with input $u_{1}$ and output $y_{2}$, the two derivatives are "hidden". It is easy to check that this temporal system is impulsively regular; in addition, one has the following CLCF: $B(\partial)=A^{-1}(\sigma) B^{+}(\sigma)$ with $A(\sigma)=$ $\operatorname{diag}\left(\sigma^{2}, \sigma^{2}, 1\right)$ and

$$
B^{+}(\sigma)=\left[\begin{array}{cccc}
-\sigma^{2} & 1+\sigma^{2} t & 0 & 0 \\
0 & 0 & 1 & -\sigma^{2} \\
0 & 1 & -1 & 0
\end{array}\right]
$$

The matrix $B^{+}(\sigma)$ is equivalent over $\boldsymbol{S}$ to $\left[\begin{array}{ll}\Sigma & 0\end{array}\right]$ with $\Sigma=\operatorname{diag}\left(1,1, \sigma^{2}\right)$, thus $M^{+} \cong \boldsymbol{S} \oplus \frac{\boldsymbol{S}}{\left(\sigma^{2}\right)}$. This impulsive system $M^{+}$is defined by the following equations:

$$
\left(1+\sigma^{2} t\right) y_{1}^{+}-\sigma^{2} u_{1}^{+}=0 ; \quad u_{2}^{+}-\sigma^{2} y_{2}^{+}=0 ; \quad y_{1}^{+}=u_{2}^{+}
$$

and $\mathscr{T}\left(M^{+}\right)=\left[v^{+}\right]_{\boldsymbol{S}}$ where

$$
\sigma^{2}\left(v^{+}\right)=0, \quad v^{+}=t y_{1}^{+}+y_{2}^{+}-u_{1}^{+} .
$$

The space $\mathscr{A}_{\infty, u}$ is the set of all elements $\tilde{v} \in \tilde{\Delta}$ such that $\sigma^{2} \tilde{v}=0$ and $\mathscr{A}_{\infty}=\left(M^{+}\right)^{*}$ is the set of all elements $\tilde{w}=\left[\begin{array}{llll}\tilde{y}_{1} & \tilde{u}_{1} & \tilde{y}_{2} & \tilde{u}_{2}\end{array}\right]^{T} \in \tilde{\Delta}^{4}$ such that $B^{+}(\sigma) \tilde{w}=0$. By (29), one may write $\tilde{v}=t \tilde{y}_{1}+\tilde{y}_{2}-\tilde{u}_{1}$, and by Proposition $39, \mathscr{A}_{\infty, u}$ is the space spanned by $\tilde{v}=\alpha_{1} \tilde{\delta}+\alpha_{0} \partial \tilde{\delta}$ as $\left(\alpha_{0}, \alpha_{1}\right)$ spans $\mathbb{R}^{2}$. The space $\mathscr{B}_{\infty, u}$ is given by the relation $\mathscr{B}_{\infty, u}=\tau^{-1} \psi \mathscr{A}_{\infty, u}$. These calculations can also be made using Theorem 42.

In this rather simple example, the space $\mathscr{B}_{\infty, u}$ can be analytically calculated. One obtains: for $t \geq 0$ in the continuous-time case ${ }^{10}$, and for $t \leq 0$ in the discrete-time one,

$$
t y_{1}(t)+y_{2}(t)-u_{1}(t)=\alpha_{1} \delta(t)+\alpha_{0} \dot{\delta}(t):=v(t)
$$

${ }^{10}$ With a mild abuse of language since the signals involved here are distributions; but as they belong to the signal space $S$, this notation can be justified. 
where $\alpha_{0}:=\partial_{0}^{0}\left(y_{1}-u_{2}\right)$ and $\alpha_{1}:=\partial_{0}^{1}\left(y_{1}-u_{2}\right)$, which is in accordance with the above result.

In the algebraic method we are proposing, the expression (29) of $\mathscr{T}\left(M^{+}\right)$and of the generator $v^{+}$of $\mathscr{T}\left(M^{+}\right)$in the generators $y_{1}^{+}, y_{2}^{+}, u_{1}^{+}$and $u_{2}^{+}$of $M^{+}$has been found using unimodular matrices, i.e. with elementary row and column operations, in a systematic way, as usual (secondary row and column operations are unnecessary [5]). This method can be computerized and then applied to large-scale systems. This seems much more difficult, if not impossible, with any analytic method.

This example illustrates the fact that, for impulsively regular time-varying temporal systems as for time-invariant ones, impulsive motions occur due to "inconsistent initial conditions" in the continuous-time case and to "inconsistent final conditions" in the discrete-time one.

\subsubsection{Non-regular example}

The following lemma will be useful:

Lemma 43. For any $a \in \boldsymbol{K}$ and any integer $n \geq 0$,

$$
a \delta^{(n)}=\sum_{i=0}^{n}(-1)^{i}\left(\begin{array}{c}
n \\
i
\end{array}\right) a^{\gamma^{i} \beta^{n}}(0) \delta^{(n-i)}
$$

Proof. Noticing that $\gamma \beta=\beta \gamma,(31)$ can be easily proved by induction. Note that in the continuous-time case, this formula is classic $([31],(\mathrm{V}, 3 ; 4))$.

Consider the following example:

$$
B(\partial)=\left[\begin{array}{ccc}
-(t+\lambda) \partial^{4} & \partial & -t \\
1 & 0 & 0
\end{array}\right]
$$

where $\lambda=0$ (resp. $\lambda=4$ ) in the continuous- (resp. discrete-) time case. By Corollary 31 , the associated temporal system is semiregular since the Smith form of $B(\partial)$ over $\boldsymbol{L}$ is $\left[\begin{array}{ll}I & 0\end{array}\right]$. The variable $w_{1}$ is discontinuous at $t=0$ due to the second row, and its 4 th order derivative in the first row generates elements of $\Delta$; the latter are annihilated or modified according to (31) by the left multiplication by $t+\lambda$, which explains that the temporal system is non-regular. By (31), this temporal system has the same impulsive behavior as the temporal system with matrix

$$
B_{1}(\partial)=\left[\begin{array}{ccc}
4 \partial^{3} & \partial & -t \\
1 & 0 & 0
\end{array}\right],
$$

which is regular; $B_{1}(\partial)$ has the following CLCF over $\boldsymbol{S}$ :

$$
B_{1}(\partial)=\left[\begin{array}{cc}
\sigma^{3} & 0 \\
0 & 1
\end{array}\right]^{-1}\left[\begin{array}{ccc}
4 & \sigma^{2} & -\sigma^{3} t \\
1 & 0 & 0
\end{array}\right] .
$$


For this "regularized temporal system", $M^{+}=\Phi^{+} \oplus \mathscr{T}\left(M^{+}\right)$where $\Phi^{+} \cong \boldsymbol{s} \boldsymbol{S}$ and $\mathscr{T}\left(M^{+}\right)=\left[v^{+}\right]_{\boldsymbol{S}}$, with

$$
\sigma^{2}\left(v^{+}\right)=0, \quad v^{+}=w_{2}^{+}-\sigma t w_{3}^{+} .
$$

Therefore, $\mathscr{B}_{\infty, u}$ is the $\Re$-subspace of $\Delta$ spanned by $\delta$ and $\dot{\delta}$.

\section{Concluding remarks}

In this paper, impulsive behaviors of "temporal systems" with constant or timevarying coefficients have been studied in a general framework which includes the continuous- and discrete-time cases.

In the existing literature, tedious calculations revealed the structure of impulsive behaviors in the case of constant coefficients. These calculations are avoided here using the key isomorphism (19), the commutative diagram (21), and the "pseudoimpulsive behavior" $\mathscr{A}_{\infty}$, whose structure is deduced by duality from the structure of a finitely generated module over the ring $\boldsymbol{S}$ (Theorem 16 and Remark 17). Theorem 19 is the main structure theorem in the case of constant coefficients; the expression of $\mathscr{B}_{\infty, u}$ was already known, but its connection with the structure of the zeros at infinity of $B(\partial)(\$ 2.1 .3)$ is now much clearer; the direct sum decomposition of $\mathscr{B}_{\infty}$ is new. Theorem 21 is new and facilitates the calculation of the impulsive behavior of an interconnected temporal system.

In the case of time-varying coefficients, the results of this paper are new. The impulsive behavior is defined only for a "semiregular temporal system", and, based on Lemma 1, a necessary and sufficient condition for a temporal system to be semiregular has been given when the coefficient ring is $\Re[t]$ (Theorem 30). However, the structure of the impulsive behavior of a temporal system is easily determined only when this temporal system is "regular" (\$5.2). Most of the results previously obtained in the case of constant coefficients are then valid, with slight modifications when necessary. The isomorphism (19) is still valid, as shown by Proposition 27, but the commutative diagram (27) replaces (21). Theorems 40 and 42, which are the generalizations of Theorems 19 and 21, respectively, are the main results. Theorem 40 completely elucidates the structure of the impulsive behavior of a regular temporal system. The theory is illustrated through two examples; the first one (in §5.3.1) shows the connection between the generation of uncontrollable impulsive motions and "temporal interconnections". The second one (in §5.3.2) shows that a regularization procedure (based on Lemma 43) can be used to calculate the impulsive behavior of a semiregular temporal system which is non-regular.

\section{References}

[1] Antoniou E. N., Vardulakis A. I. G., Karampetakis N. P.: A Spectral Characterization of the Behavior of Discrete Time AR-Representations over a Finite Time Interval. Kybernetika 34 (1998), 555-564

[2] Bourlès H.: A New Look on Poles and Zeros at Infinity in the Light of Systems Interconnection. Proc. 41st Conf. on Decision and Control. Las Vegas, Nevada (December 10-13, 2002), 2125-2130 
[3] Bourlès H.: Impulsive Behaviors of Discrete and Continuous Time Varying Systems: a Unified Approach. Proc. ECC'2003, Cambridge, UK (September 1-4, 2003)

[4] Bourlès H., Fliess M.: Finite poles and zeros of linear systems: an intrinsic approach. Internat. J. Control 68 (1997), 897-922

[5] Bourlès H., Marinescu B.: Poles and Zeros at Infinity of Linear Time-Varying Systems. IEEE Trans. on Automat. Control 44 (1999), 1981-1985

[6] Campbell S. L., Petzold L.: Canonical forms and solvable singular systems of differential equations. SIAM J. Alg. Discrete Math 4 (1983), 517-521

[7] Cohn P. M.: Free Rings and their Relations. Academic Press, 1985

[8] Fliess M.: Some Structural Properties of Generalized Linear Systems. Systems \& Control Letters 15 (1990), 391-396

[9] Fliess M., Bourlès H.: Discussing some examples of linear system interconnections. Systems \& Control Letters 27 (1996), 1-7

[10] Fliess M., Mounier H.: Tracking Control and $\pi$-Freeness of Infinite Dimensional Linear Systems. In: Dynamical Systems, Control, Coding, Computer Vision, Picci, G., Gilliam, D. S. (edts). Birkhäuser, 1999, pp. 45-68

[11] Fröhler S., Oberst U.: Continuous time-varying linear systems. Systems \& Control Letters 35 (1998), 97-110

[12] Gantmacher F. R.: The Theory of Matrices (vol. 2). Chelsea Publishing Company, 1959

[13] Geerts A. H. W. (Ton), Schumacher J. M.: Impulsive-Smooth Behavior in Multimode Systems. Part I: State-space and Polynomial Representations. Automatica 32 (1996), $747-758$

[14] Granger M., Maisonobe P.: A basic course on differential modules. In $\mathscr{D}$-modules cohérents et holonomes, Maisonobe, P., Sabbah, C. (edts). Hermann, 1993

[15] Ježek J.: Rings of skew polynomials in algebraic approach to control theory. Kybernetika 32 (1996), 63-80

[16] Karampetakis N. P.: On the construction of the forward and backward solution space of a discrete time AR-representation. Proc. IFAC World Congress, Barcelona, Spain, 2002

[17] Karampetakis N. P.: On the determination of the dimension of the solution space of a discrete time AR-representation. Proc. IFAC World Congress, Barcelona, Spain, 2002

[18] Karampetakis N. P., Vardulakis A. I. G.: On the Solution Space of Continuous Time AR Representations. Proc. ECC'93, Groningen, The Netherlands (1993), 1784-1789

[19] Lam T. Y.: Lectures on Modules and Rings. Springer Verlag, 1999

[20] Lang S.: Algebra. Springer Verlag, 2002

[21] Lewis F. L.: Descriptor Systems: Decomposition Into Forward and Backward Subsystems. IEEE Trans. on Automat. Control 29 (1984), 167-170

[22] Lewis F. L., Mertzios B. G.: On the Analysis of Discrete Linear Time-Invariant Singular Systems. IEEE Trans. on Automat. Control 35 (1990), 506-511

[23] Luenberger D. G.: Dynamic Equations in Descriptor Form. IEEE Trans. on Automat. Control 22 (1977), 312-321

[24] Luenberger D. G.: Boundary Recursion for Descriptor Variable Systems. IEEE Trans. on Automat. Control 34 (1989), 287-292

[25] Marinescu B., Bourlès H.: The Exact Model Matching Problem for Linear TimeVarying Systems: an Algebraic Approach. IEEE Trans. on Automat. Control 48 (2003), 166-169

[26] McConnel J. C., Robson J. C.: Noncommutative Noetherian Rings. American Mathematical Society, 2001

[27] Oberst U.: Multidimensional Constant Linear Systems. Acta Applicandae Mathematicae 20 (1990), 1-175 
[28] Polderman J. W., Willems J. C.: Introduction to Mathematical Systems Theory-A Behavioral Approach. Springer, 1998

[29] Rotman J. J.: An Introduction to Homological Algebra. Academic Press, 1979

[30] Rosenbrock H. H., Pugh A. C.: Contributions to a hierarchical theory of systems. Internat. J. Control 19 (1974), 845-867

[31] Schwartz L.: Théorie des distributions. Hermann, 1966

[32] Vardulakis A. I. G.: Linear Multivariable Control. Wiley, 1991

[33] Vardulakis A. I. G., Fragulis G.: Infinite Elementary Divisors of Polynomial Matrices and Impulsive Motions of Linear Homogeneous Matrix Differential Equations. Circuits Systems Signal Process 8 (1989), 57-373

[34] Vardulakis A. I. G., Limebeer D. N. J., Karkanias N.: Structure and Smith-MacMillan form of a rational matrix at infinity. Internat. J. Control 35 (1982), 701-725

[35] Verghese G. C.: Infinite-Frequency Behaviour in Generalized Dynamical Systems. Ph.D. dissertation. Electrical Engineering Department, Stanford University 1979

[36] Verghese G. C., Kailath T.: Impulsive Behavior in Dynamical System: Structure and Significance. Proc. 4th Int. Symp. on Mathematical Theory of Networks and Systems. Delft, The Netherlands 1979, pp. 162-168

[37] Vidyasagar M.: Control System Synthesis-A Factorization Approach. MIT Press, 1985

[38] Wang C.-J.: State Feedback Impulse Elimination of Linear Time-varying Singular Systems. Automatica 32 (1996), 133-136

[39] Willems J. C.: Paradigms and Puzzles in the Theory of Dynamical Systems. IEEE Trans. on Automat. Control 36 (1991), 259-294

Received November 2, 2002; revised and in final form March 5, 2004

Henri Bourlès, SATIE, Ecole Normale Supérieure de Cachan and Conservatoire National des Arts et Métiers, 61 avenue du Président Wilson, 94230 Cachan, France

Henri.Bourles@satie.ens-cachan.fr 